Keywords: morphogenesis, polarity, microscopy, multi-scale analysis, image processing, cells, tissue deformation, quantitative biology, epithelia, genetic mosaics

\title{
Quantitative methods to study epithelial morphogenesis and polarity
}

\author{
Benoit Aigouy ${ }^{1 *}$, Claudio Collinet ${ }^{1 *}$, Matthias Merkel ${ }^{2 *+}$, and Andreas Sagner ${ }^{3^{*+}}$ \\ *All authors contributed equally; authorship order is alphabetical \\ +corresponding authors (mmerkel@syr.edu, andreas.sagner@crick.ac.uk) \\ 1. IBDM - Institut de Biologie du Développement de Marseille, UMR 7288, 13288 Marseille, FRANCE \\ 2. Physics Department, Syracuse University, Syracuse, NY 13244, USA \\ 3. The Francis Crick Institute, Mill Hill Laboratory, Mill Hill London, NW7 1AA, UK
}

\begin{abstract}
Morphogenesis of an epithelial tissue emerges from the behavior of its constituent cells, including changes in shape, rearrangements and divisions. In many instances the directionality of these cellular events is controlled by the polarized distribution of specific molecular components. In recent years, our understanding of morphogenesis and polarity highly benefited from advances in genetics, microscopy and image analysis. They now make it possible to measure cellular dynamics and polarity with unprecedented precision for entire tissues throughout their development.

Here we review recent approaches to visualize and measure cell polarity and tissue morphogenesis. The review is organized like an experiment. We first discuss the choice of cell and polarity reporters and describe the use of mosaics to reveal hidden cell polarities or local morphogenetic events. Then, we outline application-specific advantages and disadvantages of different microscopy techniques and image projection algorithms. Next, we present methods to extract cell outlines in order to measure cell polarity and detect cellular events underlying morphogenesis. Finally, we bridge scales by presenting approaches to quantify the specific contribution of each cellular event to global tissue deformation.
\end{abstract}

Taken together, we provide an in-depth description of available tools and theoretical concepts to quantitatively study cell polarity and tissue morphogenesis over multiple scales. 


\section{Table of contents}

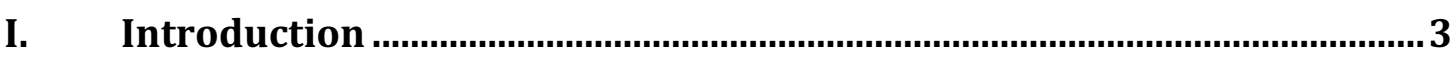

II. Biological Toolbox............................................................................

A. Cell and polarity markers ..........................................................................4

B. Mosaics as a tool to visualize polarity and morphogenesis ...........................5

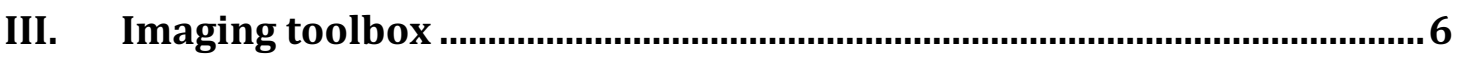

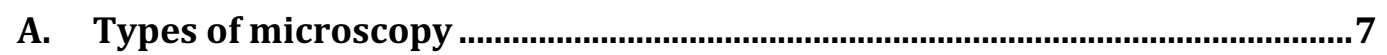

B. Tips and pitfalls to optimize imaging protocols...............................................9

1. Optimizing the dynamic range, the signal to noise ratio and the speed of imaging 9

2. Preventing photobleaching and photodamage....................................................... 9

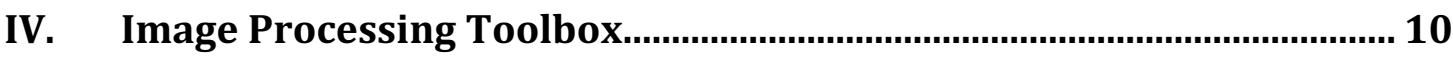

A. Projection algorithms............................................................................ 10

B. Imaging large samples with cellular resolution/Stitching ……................... 12

C. Particle Image Velocimetry ........................................................................12

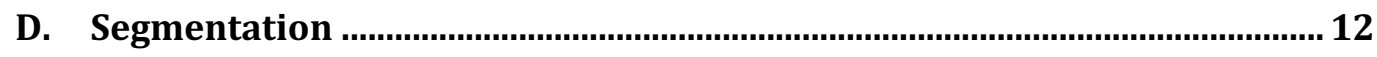

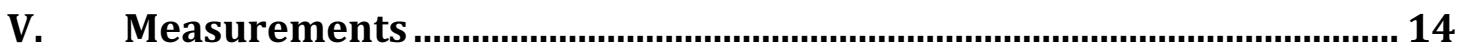

A. Planar Polarity............................................................................................ 14

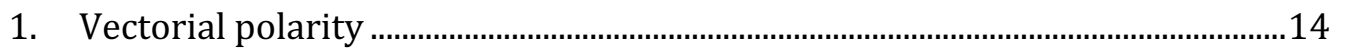

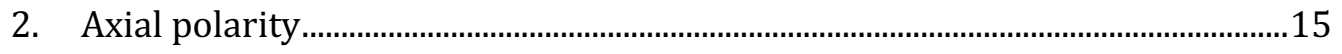

B. Tissue deformation ......................................................................................... 17

1. Without segmentation ........................................................................................17

2. With segmentation ...................................................................................................19

C. Averaging of tissue deformation and polarity patterns ............................... 20

VI. Concluding remarks ……...................................................................... 21

VII. Acknowledgement.............................................................................. 21

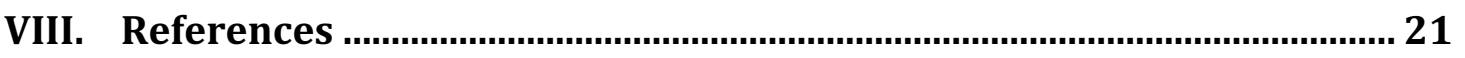




\section{Introduction}

Epithelia are the most widespread tissue in the animal kingdom. They are characterized by tight connections between cells called junctions inhibiting diffusion of molecules across the epithelium. Epithelial cells also exhibit an apico-basal polarity underlined by an asymmetric distribution of intracellular and plasma membrane components. This polarized organization allows selective and directed transport of molecules (e.g. nutrients, proteins, ions etc.) across the epithelial barrier.

Most epithelial cells exhibit a second polarity axis, called planar cell polarity (PCP) that lies within the plane of the epithelium. PCP coordinates the alignment of cell external and internal structures locally (between neighboring cells) and globally (across the tissue) (Goodrich \& Strutt, 2011). Striking examples of PCP are the coordinated alignment of hairs and cilia found in many epithelia. PCP also coordinates polarized cell rearrangements during convergence and extension movements in vertebrates and invertebrates (Bertet, Sulak, \& Lecuit, 2004; Blankenship, Backovic, Sanny, Weitz, \& Zallen, 2006; Irvine \& Wieschaus, 1994; Tada \& Kai, 2012; WalckShannon \& Hardin, 2014; Wallingford, 2012). Molecularly, PCP depends on the asymmetric segregation of polarity proteins to different sides of a cell (compare Fig. $1 \mathrm{~A}$ and $1 \mathrm{C}$ ). Therefore, in many instances PCP can be defined by a vector, which represents the asymmetric protein localization (Fig. 1A). However, several PCP components (e.g. the atypical cadherin Flamingo (Chae et al., 1999; Usui et al., 1999), Myo-II, E-cad and Par3 in the germband of Drosophila (Bertet et al., 2004; Blankenship et al., 2006; Zallen \& Wieschaus, 2004)) localize to both sides of a cell and are therefore characterized by an axial rather than a vectorial polarity (Fig. 1B).

During development, epithelia undergo dramatic morphogenetic changes to acquire their final shape. In most cases these shape changes occur without a loss of epithelial integrity and involve a limited set of cellular events occurring within the plane of the epithelium. Two classes of events can be distinguished, cell deformations and events changing the topology of the cell network (i.e. the local arrangement of cell connections). Cell deformations can be isotropic (i.e. equal changes in all directions), such as reduction or expansion of cell area, or anisotropic such as cell elongation along a specific axis. Most changes in the cell network topology result from three processes: neighbor exchanges, cell divisions and cell extrusions (Fig. 3A).

A type of neighbor exchange, called T1 transition (Bohn, 2003; Weaire \& Hutzler, 2001) is defined as the loss of contact between two cells $(a, b)$ that become separated by two adjacent cells (c,d) (Fig. 3A). T1 transitions typically involve four cells and occur in two steps: first the shrinkage of the contact between cells $a$ and $b$ followed by extension of a new contact between cells $c$ and d (Fig. 3A) (Bertet et al., 2004). In some cases more than four cells exchange their neighbors, inducing the formation of rosettes (Blankenship et al., 2006; Nishimura, Honda, \& Takeichi, 2012). When T1 transitions occur in an oriented manner throughout an epithelium, e.g. in response to PCP signaling, this results in epithelial convergence and extension. Such polarized cell intercalation has been shown to underlie germband extension in Drosophila (Bertet et al., 2004; Blankenship et al., 2006; Irvine \& Wieschaus, 1994; Walck-Shannon \& Hardin, 2014), and convergent extension in the vertebrate neural tube (Nishimura et al., 2012; Nishimura \& Takeichi, 2008), the renal tubules in mouse (Karner et al., 2009) and Xenopus (Lienkamp et al., 2012), and the mammalian cochlea (Chacon-Heszele, Ren, Reynolds, Chi, \& Chen, 2012; Wang et al., 2005). 
Cell divisions increase the number of cells, often contributing to epithelial growth, and modify network topology. Importantly, the orientation of cell divisions has been proposed to bias the orientation of growth in the Drosophila wing disc (Baena-Lopez, Baonza, \& GarciaBellido, 2005). Furthermore, oriented cell divisions have been implicated in releasing tissue level stresses as in the peripheral rim of the Drosophila wing discs (Legoff, Rouault, \& Lecuit, 2013) and during zebrafish epiboly (Campinho et al., 2013).

During cell extrusion a cell progressively loses contact with its neighbors and leaves the tissue layer. The resulting change in topology is termed T2 transition (Bohn, 2003) (Fig. 3A). Typically, they occur when cells undergo developmentally induced epithelial to mesenchymal transition (EMT) or die. Cell extrusions by $\mathrm{T} 2$ transitions constitute an important mechanism to maintain epithelial homeostasis and are observed in many epithelia, e.g. at the tip of gut microvilli in mammals (Eisenhoffer \& Rosenblatt, 2013; Guillot \& Lecuit, 2013), the developing fin of zebrafish (Eisenhoffer et al., 2012) and the midline of the developing dorsal thorax in Drosophila (Marinari et al., 2012).

In this review we provide an overview of methods to quantify morphogenesis and planar polarity at the cell and tissue scale. We first outline a selection of biological strategies to label cells and unravel polarized protein distribution. We next discuss different microscopy approaches and illustrate in detail suitable methods for image projection, segmentation, and measurement of tissue deformation and polarity. Boxes provide the necessary mathematical background for the quantification methods. At each step, we highlight potential benefits and disadvantages of the presented methods.

\section{Biological Toolbox}

Morphogenesis can be studied with different levels of detail. Below, we first describe biological markers that can be used to monitor coarse-grained tissue deformation, as well as markers to visualize single cell polarity or unravel fine-grained cellular events underlying morphogenesis. We then outline strategies using mosaics to visualize hidden polarities in epithelial tissues

\section{A. Cell and polarity markers}

In general, a good biological marker: 1) must not perturb the developmental processes studied. Ideally, it should be expressed at endogenous levels from its endogenous locus or be biologically neutral when overexpressed. 2) The marker should be bright, in particular for live imaging. Fluorescent Proteins with high quantum yield and low bleaching are preferable. According to our experience, e-GFP, Venus and td-Tomato perform better than cyan fluorescent proteins. 3) The marker should be as much as possible restricted to and label only the structure of interest (e.g. cell membrane, nucleus, etc.).

Coarse-grained analysis of tissue deformation can be performed by a method called "particle image velocimetry" (PIV) (see section IV.C) (Raffel, 2007). PIV exploits the natural heterogeneity of the tissue and works with most conventional markers (e.g. cell membrane, nuclei, organelles, etc.). 
For a fine-grained analysis of cellular events underlying morphogenesis, an extraction of cell outlines is often required (i.e. segmentation). Optimal reporters for this display a sharp signal around the entire cell perimeter and have low or no cytoplasmic interfering signal (such as vesicles). E-cadherin::e-GFP is such a marker for Drosophila tissues. Discontinuous or less sharp markers can also be used to extract cell outlines (e.g. many planar polarity proteins and components of the cytoskeleton), but with poorer results. Note that to study the distribution of such proteins in the cell, cell outlines may be obtained with a second, more easily segmentable marker (e.g. E-cadherin::e-GFP) and then applied to the signal of the former (planar polarity proteins cytoskeletal components etc.).

\section{[[[ Desired position of Figure 1: somewhere within the section "Mosaics as a tool to visualize polarity and morphogenesis" ]]]}

\section{B. Mosaics as a tool to visualize polarity and morphogenesis}

The limited resolution of conventional light microscopy cannot resolve the relative protein contributions of adjacent cells to their shared boundary. Therefore, a protein localizing only to one side of the cell may appear bipolar or non polarized (Fig. 1E,F,I,K). Hence, vectorial polarity can be hidden (Fig. $1 \mathrm{l}, \mathrm{K}$ and section V.A.2), although in many situations, an axial polarity can still be detected (Fig. 1D-F).

Genetic mosaics, i.e. tissues consisting of two or more genetically distinct cell populations, provide an elegant solution to this problem, because they allow the expression of a labeled protein in only a subset of cells (Fig. $1 \mathrm{G}, \mathrm{H}, \mathrm{L}$ ). As a consequence, any labeled protein localized at the interface between labeled and unlabeled cells comes only from the labeled cells. Thus, this interface can be used for the quantification of magnitude and direction of PCP vectors (Fig. 1D$M$ and section V.A.1). Note however that there may be rare cases where vectorial polarity can be measured without requiring mosaics, for example when few cells show higher fluorescence intensity than their neighbors (Besson et al., 2015).

Mosaics can be induced in several ways in several organisms. They can be created by injecting, infecting or transfecting cells with vectors or by creating chimeras between different animals (Kretzschmar \& Watt, 2012). However, the two most widely used methods are the Flp/FRT and the Cre/lox systems (Nagy, 2000; Sauer \& Henderson, 1988; Xu \& Rubin, 1993). In both cases, the expression of a recombinase (Flippase/Flp or Cre) induces recombination between two target DNA sequences (FRT or loxP sites). Furthermore, both systems are highly versatile as they allow tight regulation of recombinase activity in time and space (Danielian, Muccino, Rowitch, Michael, \& McMahon, 1998; Hayashi \& McMahon, 2002; Xu \& Rubin, 1993). Recombination in cis (i.e. between sites on a single DNA molecule) or in trans (on different molecules) can be mediated using both systems. When two recombination sites flank a DNA sequence, recombination in cis results in the excision of the flanked DNA fragment. This is often used to delete parts of a gene to mutate it or to excise a central "Stop" cassette to conditionally activate gene expression (Kretzschmar \& Watt, 2012; Srinivas et al., 2001). This for example allows the generation of multiple small labeled clones in an otherwise unlabeled tissue. In Drosophila, recombination in trans between two FRT sites is used to exchange distal arms of 
homologous chromosomes during mitosis (Golic, 1991; Xu \& Rubin, 1993). Such a recombination can generate two genetically distinct daughter cells from a heterozygous mother cell.

Genetic mosaics can also be used to label clonally related cells to visualize local morphogenesis. Indeed, clone shape results from all the cellular events occurring within the clone. For example, elongated clone shape indicates anisotropic cell behaviors (e.g. oriented cell divisions, oriented neighbor exchanges, and/or cell elongation), while round clones may result from either absence of anisotropies or opposing anisotropic cellular behaviors. Similarly, clone fragmentation indicates a high rate of neighbor exchange. However, absence of clone fragmentation does not necessarily indicate absence of neighbor exchanges. This is the case in the Drosophila wing, where clonally related cells mostly remain attached to each other despite extensive cell rearrangements (Aigouy et al., 2010; Heller et al., 2016). Additionally, reproducible differences in clone size indicate heterogeneous growth within the tissue. Also, obtaining few snapshots of the development of clones allows to observe their deformation as well as to infer global tissue deformation by triangulation between them (Heemskerk, Lecuit, \& LeGoff, 2014).

\section{Imaging toolbox}

Imaging (i.e. the acquisition of images with a microscope) is an essential technique to understand the cell biological and molecular basis of development. Light microscopy allows to observe tissues with cellular and subcellular resolution. Since morphogenesis is a dynamic process that is better understood when followed in real time, live imaging is the primary approach to study morphogenesis in many labs. Furthermore, although cell and tissue polarity can be studied in fixed tissues, understanding how polarity is established and maintained in face of dynamic cell behaviors greatly benefits from live imaging.

A microscopy image is nothing but a visual representation of a 2 dimensional $(x, y)$ matrix of intensity values (Fig. 2A). Each element of this matrix is called a pixel. Pixels can be coded over $n$ number of bits, typically 8 to 16 for most biological images. Pixel intensities in 8 bit images therefore range from 0 to 255 (i.e. $2^{8}$ gray levels) and in 16 bit images from 0 to 65535 (i.e. $2^{16}$ gray levels). Imaging datasets of labeled biological samples can have more than just 2 dimensions when also the sample depth ( $z$ dimension), time (t dimension) and multiple colors (c dimension) are considered. In this section we present the most common types of microscopy and discuss their applications, advantages and disadvantages relatively to different biological questions (Table 1).

Table 1: Comparison of different types of microscopy.

\begin{tabular}{|l|l|l|l|l|}
\hline $\begin{array}{l}\text { Microscope/type } \\
\text { of analysis }\end{array}$ & Polarity & $\begin{array}{l}\text { Tissue scale } \\
\text { morphogenesis }\end{array}$ & $\begin{array}{l}\text { Cell scale } \\
\text { morphogenesis }\end{array}$ & Phototoxicity* \\
\hline Widefield & + & +++ & + & + \\
\hline Confocal & +++ & + & +++ & ++ \\
\hline Spinning disk & +++ & +++ & +++ & + \\
\hline
\end{tabular}




\begin{tabular}{|l|l|l|l|l|}
\hline $\begin{array}{l}\text { Light sheet } \\
\text { microscope }\end{array}$ & + & +++ & +++ & + \\
\hline $\begin{array}{l}\text { Two-photon } \\
\text { microscopy }\end{array}$ & +++ & + & +++ & +++ \\
\hline
\end{tabular}

*more "+" means more phototoxic

\section{A. Types of microscopy}

The simplest and cheapest microscopy set-up is widefield imaging (also called epifluorescence). An epifluorescence microscope captures the light emitted by the whole sample with an objective lens and records each image at once with a CCD camera. Widefield imaging is therefore fast but it also collects out-of-focus light from regions above and below the focal plane. While this is generally not a problem when imaging thin samples (e.g. cells in a petri dish), the out-of-focus light reduces the contrast in images of whole mount organisms.

This limitation is overcome in confocal microscopy, which allows to acquire thin optical sections to obtain sharp 3D representations of the sample. Laser scanning confocal microscopy (LSCM) and spinning disk confocal microscopy (SDCM) are the two most commonly used types of confocal systems. They both utilize pinholes, i.e. apertures in the detection path that reject out-of-focus light generating images with higher contrast compared to widefield. LSCM and SDCM differ in how the sample is scanned and signals are detected, making the two systems optimal for different applications.

In LSCM a laser is moved with mirrors across the sample and the image is generated pixelby-pixel by recording the emitted photons using a photon-multiplying tube (PMT). The PMT converts the photons into electrical/analog signals stored as intensity values in an image by the recording software. LSCM offers good $x, y$, and z-resolution close to the theoretical limit of conventional light microscopy and a high signal-to-noise ratio $(\mathrm{S} / \mathrm{N})$. Properly adjusting the scan speed parameter, i.e. the time the laser spends on each pixel (also referred to as pixel dwell time), allows to improve $\mathrm{S} / \mathrm{N}$ and therefore the quality of the acquired images. Traditionally, the main drawback of LSCM is the overall low speed of acquisition, especially when multiple channels, large $(x, y)$ fields and big z-volumes need to be recorded. Nevertheless, modern LSCM systems can be used to perform relatively fast live imaging. Alternatively, scan speed can be increased by reducing the field of view or at the expense of S/N. Taken together, LSCM is an excellent choice for acquiring images with high resolution and good $S / N$, when slow acquisition is not a problem. Indeed, LSCM is most often the prime choice for fixed samples stained by immunofluorescence.

A recent development in LSCM is the use of multi-photon excitation (most frequently two-photon on conventional systems) (Denk, Strickler, \& Webb, 1990). Classically, fluorophores are excited by a single photon with a wavelength shorter than that of the emitted photon. In contrast, with multi-photon excitation, the fluorophore is excited by simultaneous absorption of two or more photons with a larger wavelength (multi-photon effect). This offers three main advantages compared to classical LSCM. First, photons with longer wavelengths can penetrate further into the sample and thereby allow deeper imaging (Helmchen \& Denk, 2005). Second, since the photon density required for the multi-photon effect is reached only at the focal plane, all emitted photons are "in-focus" and there is no need for pinholes to filter out-of-focus light. 
This dramatically increases the $\mathrm{S} / \mathrm{N}$ giving this set-up an advantage when imaging light scattering samples (Denk \& Svoboda, 1997). Third, because fluorophores are only excited in the focal plane and not, as in classical LSCM, throughout the sample, multi-photon microscopy causes less photobleaching, especially when acquiring large z-volumes (So, Dong, Masters, \& Berland, 2000). However, multi-photon imaging also has disadvantages. First, since the scanning principle is similar to regular LSCM, multi-photon imaging is slow. Additionally, it is not well suited for multicolor imaging because multi-photon lasers usually need time-consuming steps of tuning to switch between different wavelengths and cannot be easily combined with visible lasers for regular LSCM. Finally, the high photon density of multi-photon lasers can cause photodamage and in many cases is not well tolerated by the sample. In conclusion, multi-photon microscopy provides a good alternative to regular LSCM when deep sample penetration is required.

Spinning disk confocal microscopy (SDCM) has emerged in the recent years as a powerful technique to perform fast imaging of living samples. In SDCM, instead of using a single pinhole, a rotating disk with several thousand pinholes (also called Nipkow disk) is put into the conjugate image plane. In a recent development of SDCM (the Yokogawa spinning disk unit) the illumination beam is focused on the spinning disk that effectively multiplies it into thousands of parallel beams focused onto the sample by the objective lens. Upon disk rotation the parallel beams simultaneously scan the entire specimen at a speed that is much higher than LSCM. The emitted light coming from the sample is collected back through the pinholes producing an optical section that is registered at once with an image by CCD cameras. Such a parallel scanning and reading strategy requires low sample illumination and thereby reduces photobleaching and photodamage. Thus, SDCM is a good choice to image living samples and fast developmental processes. Also this is an excellent device to image large samples with cellular resolution using image stitching (see section IV.B). Disadvantages of this setup are that the size of the pinholes in the spinning disk is fixed and usually adjusted to yield optimal results with a certain objective (usually $40 x$ or $100 x$ ). Thus, when imaging with other magnifications, the size of the pinholes in the disk does not provide an optimal photon yield and z-resolution. In summary, spinning disk microscopes are a good choice for imaging fast developmental processes and/or entire tissues with cellular resolution.

Recently, selective plane illumination microscopy (SPIM) has emerged as an interesting technique to study developmental processes. In SPIM, the specimen is illuminated with a thin light sheet and the emitted photons are collected in perpendicular direction (Keller \& Stelzer, 2008; Weber \& Huisken, 2011). SPIM has two main advantages. First, optical sectioning is produced by illuminating only the desired planes rather than rejecting out-of-focus light with pinholes. This dramatically reduces photobleaching and photodamage allowing long-term imaging of living samples. Second, since the entire $(x, y)$ plane is illuminated at once, this technique allows fast 3D scanning of the specimen with sensitive high-speed CCD cameras. By embedding the sample in a rigid transparent medium (e.g. agarose), it is possible to rotate it and collect 3D stacks at different angles, providing isotropic resolution in the $x, y$, and $z$ dimension and allowing in toto 3D imaging of living specimens (Huisken \& Stainier, 2009; Weber \& Huisken, 2011). This technique may however produce light scattering artifacts in opaque specimens (e.g. shading due to opaque objects). Recent development including dual-side illumination with multidirectional selective plane illumination (mSPIM) have been shown to significantly alleviate these problems (de Medeiros et al., 2015; Huisken \& Stainier, 2007; Krzic, Gunther, Saunders, Streichan, \& Hufnagel, 2012). Finally, since SPIM can produce large datasets, 3D reconstruction 
can be long and computationally intensive. In conclusion SPIM is currently limited by difficulties in data handling and storing but remains a promising technique for fast 3-dimensional and multi-view imaging of biological samples with very low photobleaching.

\section{B. Tips and pitfalls to optimize imaging protocols}

Microscopy images have to respect certain criteria to provide meaningful data quantifications. In this section we discuss potential tips and pitfalls to optimize imaging protocols and acquire images appropriate for image analysis.

\section{Optimizing the dynamic range, the signal to noise ratio and the speed of imaging}

A good image is an image that: 1) uses the full dynamic range provided by the bit depth ( 8 to 16-bit), 2) in which pixels neither reach the maximum nor the minimum value of such range 3) has a good $\mathrm{S} / \mathrm{N}$ ratio. In fluorescence microscopy this can be achieved by increasing the power of the illuminating laser. This generates higher signals and improves the $\mathrm{S} / \mathrm{N}$ ratio without compromising on imaging speed. The major downside of using high laser power is photobleaching and photodamage (see next section). Usually, appropriate illumination power settings are those that maximize $\mathrm{S} / \mathrm{N}$ while minimizing photobleaching.

When increasing laser power is detrimental for the sample, other parameters controlling photon detection or photon conversion into digital signals can be tuned to improve image quality. In epifluorescent, SDCM and SPIM the amount of collected signal depends on the camera exposure time (i.e. the total time the camera spends collecting photons). Increasing exposure time yields brighter images with better $\mathrm{S} / \mathrm{N}$ at the expense of imaging speed. It is important, especially for live imaging, to find the right trade-off between exposure time and image quality. In LSCM, detection can be regulated by controlling the pixel dwell time (equivalent of exposure time) or the pinhole aperture. $\mathrm{S} / \mathrm{N}$ can be increased by either decreasing the scan speed (i.e. increasing the dwell time) or by averaging multiple scans. Of note, in LSCM, following an appropriate phase correction, dual scanning (scans of sequential lines in a leftright-left manner) can be activated to reduce the scan time while keeping dwell time constant. This option is especially useful for live cell imaging. Furthermore, when the emitted fluorescence spectra do not overlap, simultaneous detection of multiple fluorophores can be used to avoid compromising on acquisition speed. Finally, differently from SDCM, in LSCM the aperture of the pinholes can be adjusted allowing to increase the thickness of the optical slice (i.e. decreases confocality) thereby collecting more photons without decreasing imaging speed.

The gain parameter controls the electron amplification process. Upon increase higher signals are obtained from the same amount of photons collected. This allows a better use of the dynamic range, but also introduces more noise, having a moderate effect on image quality.

\section{Preventing photobleaching and photodamage}

Common problems in live imaging are photobleaching and sample photodamage. Photobleaching is the gradual loss of emitted fluorescence when labeled samples are 
illuminated with a laser. This is due to the photochemical alteration of fluorophores resulting in the permanent loss of the ability to emit photons. Photobleaching is one of the first signs that a sample is either illuminated for too long or with too high laser intensity. Although photobleaching can be corrected a posteriori, it is preferable to reduce the intensity of the exciting laser or exposure of the sample to light whenever photobleaching is detected.

Light can also damage the sample due to the generation of toxic side products (usually reactive oxygen species). Photodamage can lead to slower or abnormal development. Comparisons to fixed or living samples not subjected to the imaging protocol at comparable stages of development is usually sufficient to detect photodamage. Photodamage is therefore an important factor to be taken into consideration when designing live imaging protocols especially when studying long morphogenetic processes.

Altogether, the choice of the imaging approach depends on the biological system and the question addressed, and proper tuning of imaging parameters are key to obtain high quality images for analysis while preserving sample health.

\section{[[[ Desired position of Figure 2: somewhere within the section "Image Processing Toolbox"]]]}

\section{Image Processing Toolbox}

In this toolbox we present tools to project 3D stacks into 2D images and create large views of tissues by combining small high resolution views. Next we describe algorithms to follow and measure cell and tissue movements with or without segmentation.

\section{A. Projection algorithms}

Although often imaged with 3D stacks, epithelia are well approximated in 2D. Hence, image analysis is usually performed in 2D images. Such images are obtained by converting the three-dimensional information contained in a $\left(x\left\llcorner, \mathrm{y} \_\right)\right.$stack into a single two-dimensional $(\mathrm{x}\llcorner\mathrm{y})$ image, through a process called a projection. Numerous projection algorithms are available, but according to our experience, some are better suited than others for segmentation and polarity quantification (Table 2).

Table 2: Comparison of different 2D projection algorithms.

\begin{tabular}{|l|l|l|l|}
\hline $\begin{array}{l}\text { Projection } \\
\text { algorithms/Experimen } \\
\text { ts }\end{array}$ & Polarity & $\begin{array}{l}\text { Tissue scale } \\
\text { morphogenesis }\end{array}$ & $\begin{array}{l}\text { Cell scale } \\
\text { morphogenesis }\end{array}$ \\
\hline Maximum & ++ & ++ & ++ \\
\hline Average & +++ & +++ & + \\
\hline Stack focuser & + & + & +++ \\
\hline
\end{tabular}




\begin{tabular}{|l|l|l|l|}
\hline $\begin{array}{l}\text { Merkel et al. } 2014 \\
\text { (M. Merkel et al., } \\
\text { 2014) }\end{array}$ & ++ & + & +++ \\
\hline $\begin{array}{l}\text { Extended Depth of } \\
\text { field }\end{array}$ & + & + & ++ \\
\hline
\end{tabular}

The most widespread projection algorithm is the maximum (Max) projection. It is a pixelbased algorithm that identifies the pixel at position $\left(x_{p}, y_{p}\right)$ with the maximum intensity value along the Z-axis of the stack (Fig. 2B,D). This brightest pixel is then used in the 2D projection image. Max projection works particularly well when the signal is spatially restricted, especially in the $z$ direction, as for most junctional markers. However, by construction Max projection gathers noise from every focal plane, often yielding a noisy image when the input is noisy.

Another useful pixel-based algorithm is the average projection. This algorithm computes for every pixel at position $\left(x_{p}, y_{p}\right)$ the average pixel intensity over all $z$ planes (Fig. 2B,D). This average is then assigned to the pixel at position $\left(x_{p}, y_{p}\right)$ in the 2D projection (Fig. 2B,D). In contrast to max projection, the average projection does not discard any signal. However, average projections often show poorer contrast than other algorithms, especially for large zstacks when the signal of interest is confined to few planes. Although average projection is not the best choice for segmentation, it remains the preferred algorithm for polarity quantification.

Often, max projection is sufficient to easily segment images. However, when this is not the case, we recommend using one of the "focusing" projection algorithms described below. These algorithms assign to each region of the projection the signal from the most focused zplane at this position. This usually enhances image contrast and hence simplifies image segmentation. The extraction of in-focus signals can be done pixel by pixel, using the "extended depth of field" ImageJ/FIJ plugin (Fig. 2D) (Forster, Van De Ville, Berent, Sage, \& Unser, 2004; Preibisch, Saalfeld, \& Tomancak, 2009; Schindelin et al., 2012; Schneider, Rasband, \& Eliceiri, 2012), or for image blocks of defined width and height (Fig. 2C). "Stack focuser" is an efficient block-based algorithm that runs an edge detection filter on each z-plane of the block. The zplane presenting the sharpest edges is used for the $2 \mathrm{D}$ projection. The major drawback of this algorithm is that the sharpest signal is sometimes not the signal of interest. This can give rise to projection artifacts, where neighboring blocks carry signal coming from different depths with an intensity mismatch (Fig. 2D'). Such block artifacts can be corrected by ensuring that the selected z-planes of adjacent blocks have similar depths. This correction is usually achieved using surface smoothing algorithms (Heller et al., 2016; Legoff et al., 2013; M. Merkel et al., 2014). This correction can be combined with a max projection of a few planes surrounding the best-focused plane to further attenuate block artifacts (M. Merkel et al., 2014). Of note, block-based projections are by construction sensitive to sample curvature and can give different results depending on chosen block size. Consequently, block-based projections are not optimal for polarity measurements.

Altogether, some projection algorithms are optimized for polarity measurements (e.g. average projection) while others are better suited for segmentation (e.g. max projection). Note, however that images can first be projected with one algorithm and the resulting segmentation mask (see section D) can be applied to a different projection of the same image for measurement (see section $\mathrm{V}$ ). 


\section{B. Imaging large samples with cellular resolution/Stitching}

Studying morphogenesis or polarity greatly benefits from imaging with cellular resolution (see section V). However, high resolution is obtained at the expense of the size of the field of view. Hence, with conventional microscopes it is not possible to capture an entire tissue with cellular resolution. This drawback can be overcome by moving the sample to acquire a mosaic of several overlapping $x, y, z$ stacks (i.e. tiles) that together cover the entire tissue. Bringing those tiles together into a single image of the tissue is called stitching. In practice, stitching algorithms identify features within stacks used as landmarks. Matching these landmarks between adjacent tiles allows to compute the $x, y, z$ translation required for their alignment. Some rules need to be observed in order to obtain efficient stitching: 1) Neighboring tiles must slightly overlap (according to our experience, 5 to $10 \%$ overlap is sufficient) and 2) The time difference between the acquisitions of overlapping tiles should be sufficiently small to preserve landmark correspondence. We have obtained best results using the highly flexible "Grid/Collection stitching" FIJI plugin (Preibisch et al., 2009).

\section{Particle Image Velocimetry}

PIV (particle image velocimetry) allows to visualize the relative speed of displacement of different regions in a tissue and can be used to measure tissue deformation (see also section V.B.1). PIV was initially designed to study fluid dynamics by adding small (non-perturbing) tracer particles to the fluid (Raffel, 2007). Following the displacement of the particles allows to characterize the fluid's flow field. This technique can be directly applied to study subcellular, cellular or tissue motion upon injection of fluorescent beads or by using the natural heterogeneities of fluorescently labeled images (Aigouy et al., 2010; Collinet, Rauzi, Lenne, \& Lecuit, 2015; Ganguly, Williams, Palacios, \& Goldstein, 2012; He, Doubrovinski, Polyakov, \& Wieschaus, 2014; Levayer \& Lecuit, 2013; Mayer, Depken, Bois, Julicher, \& Grill, 2010; M. Merkel et al., 2014; Supatto et al., 2005). PIV divides the image into rectangular blocks and tries to infer, how much and in which direction each of these blocks displace from one frame to the next. As a measure of block similarity, spatial cross correlations between pixel intensities are computed across consecutive frames. Knowing the displacement of all blocks over time allows to calculate the flow field of the sample. PIV works for a broad range of tissues irrespective of the staining used (e.g. cell membranes, nuclei, intracellular organelles, etc.) and is fast because it does not require segmentation. Several PIV implementations are available in ImageJ/FIJI and Matlab (i.e. MatPIV by J. K. Sveen).

\section{Segmentation}

PIV provides rapid quantification of tissue movements, but is not sufficient to deeply characterize the cellular events at the basis of morphogenesis. To access this information, images need to be segmented. Here, we focus on algorithms to segment membrane-labeled cells.

Segmentation is nothing but a simplification process. For our purpose segmentation means assigning image pixels to two categories, 1) the boundary pixels and 2) the cytoplasmic pixels. The watershed segmentation is often used for that (Vincent \& Soille, 1991). In the 
watershed representation, the $2 \mathrm{D}$ image is seen as a landscape; regions with high pixel intensities (in our case, cell membranes) are mountains and regions with low pixel intensities (in our case, cell cytoplasm) are valleys. Immersing this virtual landscape in water will create one basin per valley. Each basin is assigned a unique identity represented by a color. Raising the water level expands individual basins until adjacent basins meet. When this occurs, a so-called watershed line is drawn between them. This watershed line labels the cell outline while the colored basin corresponds to the cell cytoplasm (Fig. 2E). There are several implementations of the watershed algorithm available for ImageJ and Matlab. In FIJI/ImageJ, we recommend using the "graylevel watershed" plugin (http://bigwww.epfl.ch/sage/soft/watershed/) rather than the default implementation. Blurring images is often useful before using the watershed algorithm, as noise leads to oversegmenation (i.e. too many cells detected). However, in order to preserve cell outlines it is important to use so-called "edge preserving filters" (such as Gaussian, bilateral, anisotropic diffusion or Kuwahara filter) (Pal, Chakrabarti, \& Ghosh, 2015).

Many cellular properties can be directly extracted from the watershed segmentation mask. Cell area, for example, corresponds to the number of cytoplasmic pixels of a cell plus half of the number of perimeter pixels (since cell outline is shared with adjacent cells). The length of the cell perimeter (in pixels) is obtained by summing the distances between centers of adjacent cell outline pixels (Fig. 2F). A vertical or horizontal connection is 1 pixel long and an oblique connection has a length equal to $\sqrt{2}$ (Fig. 2F). Cell perimeter or area in pixels can be converted to real units by multiplying them by the pixel size or the square of the pixel size in real units, respectively. The $x$ and $y$ coordinates of the cell center are obtained by averaging the $x$ and $y$ coordinates of all the pixels of the cell cytoplasm, respectively. Cell elongation, i.e. cell shape anisotropy, has been quantified in different ways (Aigouy et al., 2010; Blanchard et al., 2009; Brodland, Chen, \& Veldhuis, 2006; Etournay et al., 2015; Graner, Dollet, Raufaste, \& Marmottant, 2008; Guirao et al., 2015; Lynch, Veldhuis, Brodland, \& Hutson, 2014). One widespread method first fits an ellipse to the cell outline or cytoplasm pixels. Then, cell elongation is defined as the ratio between the lengths of the long and short axes of the ellipse. The orientation of cell elongation is given by the orientation of the long axis of the ellipse. Note however that there are different ways to fit an ellipse to a set of pixels, which might give slightly different elongation and orientation (Stojmenovic \& Nayak, 2007).

To extract topological properties such as neighbor number or to identify individual cellcell contacts, further segmentation of the watershed mask is required. To identify vertices (i.e. the point of contact between three or more cells) and bonds (i.e. points of contact between exactly two cells), a $3 \times 3$ pixel mask needs to be slid over every pixel of the cell outline. If the central pixel is surrounded by exactly two colored basins, then the pixel belongs to a boundary (Fig. 2G). In contrast, if this pixel is surrounded by three (or more) different basins then it is a vertex (Fig. 2G). The length of these newly identified cell-cell contacts can be measured as previously described for the perimeter, the first and the last pixels of the contact being the vertices. Finally, cell neighbor number can be obtained by counting the number of vertices along the cell perimeter.

Further information can be extracted by tracking cells. Tracking cells in a time-lapse movie means assigning unique identities to cells in the field of view and consistently re-identifying these cells over subsequent frames. Re-identification is usually based on cell position, cell neighborhood and/or on cellular features such as cell area or shape. Dividing and extruding cells can be found by analyzing appearance or disappearance of cell tracks. For example, cell division 
can be detected as the sudden appearance of two new cells whose combined area is similar to that of the mother cell prior to division. A T2 transition is detected as a cell disappearing from the field of view. Note that in all cases, cells entering or leaving the field of view from the sides should not be considered as dividing or dying. A T1 transition is defined as a neighbor exchange involving four adjacent cells (Fig. 3A). To detect occurring T1 events, it is necessary to identify for each cell-cell junction in the movie the pairs of cells sharing ( $a$ and $b$ ) or separated (c and d) by this junction (Fig. 3A). When in a subsequent frame, cells $a$ and $b$ are separated by a junction between cells $\mathrm{c}$ and d, a T1 transition occurred (Fig. 3A). A rosette is an exchange of neighbors between five or more cells. It is characterized by the concomitant shrinkage of two or more adjacent contacts to a single point prior to the resolution of the rosette. The simplest way to detect a rosette is to detect two or more adjacent contacts with a similar orientation having a combined length inferior to a user-defined threshold (Blankenship et al., 2006).

Note that several free software suites are available to segment and analyze epithelial cells. They conveniently combine in a single tool segmentation algorithms, cell feature extraction and, in some case, even allow to track cells (Aigouy et al., 2010; Cilla et al., 2015; Etournay et al., 2016; Gelbart et al., 2012; Heller et al., 2016; Khan, Wang, Wieschaus, \& Kaschube, 2014; Leung \& Fernandez-Gonzalez, 2015; Mosaliganti, Noche, Xiong, Swinburne, \& Megason, 2012; Sagner et al., 2012).

\section{Measurements}

\section{A. Planar Polarity}

In this section, we discuss different segmentation-based methods to quantify vectorial and axial polarity. In general, methods based on segmentation are more precise, but also more labor intensive.

\section{Vectorial polarity}

For light microscopy images, membrane-associated vectorial polarity should be measured on mosaic stainings (see section II.B). Several approaches have been used to quantify polarity vectors for a connected group of labeled cells in a mosaic tissue (Ambegaonkar \& Irvine, 2015; Brittle, Thomas, \& Strutt, 2012; Devenport, Oristian, Heller, \& Fuchs, 2011; M. Merkel et al., 2014; Sagner et al., 2012), which for the sake of simplicity we call "clone" throughout this section. Here, we present two methods to measure polarity of single cells and one method to measure the polarity of "clones".

Besson et al. (Besson et al., 2015) define the polarity vector of a single cell from the angular distribution of pixel intensities along the cell outline (Fig. 1 $R^{\prime}$ ). The polarity vector of the cell is defined as the sum of polarity vectors for pixels along the cell outline. Each pixel vector is computed as an outward directed vector, which points from the cell center to the pixel itself, and has a length equal to the product between the pixel intensity and its distance from the cell center. To include most of the signal at cell-cell junctions, the segmentation mask can be expanded in width. Besson et al. normalize the polarity vector of a cell by the product between 
the total cell outline intensity and an average cell radius. This allows to compare cell polarity vectors among different cells.

By construction, this method is sensitive to cell shape. In particular, a cell with a homogeneous signal along its outline may appear polarized when asymmetrically shaped. To overcome this shape dependency, we propose a modified version of the method described above. In this version, the length of each pixel vector is the product between the pixel intensity and the angle interval between its two neighbors along the outline (see next section and Fig. 1P). Also, we propose to normalize by the sum of all vector lengths. As a consequence, a cell with constant pixel intensity along its outline will have a polarity vector of zero.

To measure the polarity vector of "clones" of cells, Sagner et al. use the segmented "clone" outline (Sagner et al., 2012). The "clone" polarity vector (thick yellow arrow) is the sum of polarity vectors (green arrows) of each cell-cell interface along the "clone" outline (Fig. $\left.1 Q, S, S^{\prime}\right)$. Each interface polarity vector is an outward pointing vector with a direction perpendicular to the interface (defined by the straight line connecting its two vertices, blue lines in Fig $1 S^{\prime}$ ) and a length equal to the sum of pixel intensities along the segmentation mask. To compare samples with different overall intensities, the "clone" polarity vector can be normalized by the total outline intensity. As a result, the length of the normalized "clone" polarity vector varies between zero (i.e. the "clone" is not polarized) and one (i.e. all non-zero interface vectors point in the same direction).

Finally, in cases where polarity markers are clearly distinguishable from the cell membrane, neither clonal staining nor segmentation is needed to determine vectorial cell polarity. In such a case, the location of the signal of interest with respect to a reference signal (for instance the cell nucleus or the cell membrane) can be quantified using so-called "fluorescence cross-correlations" (Matis, Axelrod, \& Galic, 2012).

\section{Axial polarity}

Several methods to quantify axial polarity are available (Aigouy et al., 2010; F Bosveld et al., 2012; Floris Bosveld et al., 2016; Matthias Merkel, 2014; M. Merkel et al., 2014; Rezakhaniha et al., 2012, Sagner et al., 2012). Here we focus on two methods that require segmentation. The first method (Blankenship et al., 2006; Levayer, Pelissier-Monier, \& Lecuit, 2011; Marcinkevicius, Fernandez-Gonzalez, \& Zallen, 2009; Rauzi, Verant, Lecuit, \& Lenne, 2008; Simoes Sde et al., 2010; Tamada, Farrell, \& Zallen, 2012) measures the relative protein concentration at junctions as a function of their orientation. Practically, for each junction the average intensity and angle relative to a reference axis (e.g. antero-posterior, proximal-distal etc.) are measured. Junctions are assigned to different angular bins and their intensities are averaged. The dominant axis (or axes) of polarity is revealed by plotting average intensities as a function of angle in a histogram. To compare data from different samples, the junctional intensities can be normalized by the average of all interface intensities within each image. To increase the dynamic range of the measured polarity, camera and cytoplasmic background can be subtracted before intensity measurements (Collinet et al., 2015; Heemskerk et al., 2014; Munjal, Philippe, Munro, \& Lecuit, 2015; Simoes Sde et al., 2010; Tamada et al., 2012). Note that, although this method may appear local, because it uses cell-cell interfaces, the local information is lost in the binning process and the method rather provides the axial polarity of the whole tissue. This method has often been applied to measure polarity in the germband of Drosophila using angular bins 
between zero and 90 degrees (Levayer et al., 2011; Rauzi et al., 2008; Simoes Sde et al., 2010; Tamada et al., 2012). We recommend, instead, binning between 0 and 180 in order to distinguish not only polarities with horizontal and vertical orientations (i.e. enrichment at interfaces with 0 or 90 degrees), but also those with diagonal orientations (i.e. enrichment on interfaces with 45 and 135 degrees) (Blankenship et al., 2006). A related, but segmentationindependent, method is provided by the tool "OrientationJ". This uses an edge detection formula and displays the local edge orientation with an angle-dependent color (Matis, RusslerGermain, Hu, Tomlin, \& Axelrod, 2014; Rezakhaniha et al., 2012).

As an alternative to an angular intensity plot, axial polarity can also be characterized using a mathematical object called "nematic" (polarity box). A nematic is composed of an orientation and a magnitude, much like a vector. The main difference is that the orientation of a nematic merely defines an axis, while the orientation of a vector defines an axis and "points into" one of the two possible directions (Figs. 1A,B). Throughout this manuscript, we will denote nematics by bold symbols with a tilde: $\widetilde{A}$.

The axial polarity of a cell can be quantified by a nematic $\widetilde{A}$ similarly to the method illustrated above for vectorial polarity of a cell (Aigouy et al., 2010; M. Merkel et al., 2014; Sagner et al., 2012). The cell polarity $\widetilde{A}$ is computed as the sum of nematics defined for each pixel of the cell outline (Fig. 1P). The axis of the pixel nematic is obtained by connecting the pixel to the cell center (green line, pixel marked by magenta outline, Fig. 1P). The magnitude of the pixel nematic is a product between the pixel intensity and the angle interval between its two neighboring pixels (angle marked in red in Fig. 1P). Like for the vectorial method discussed above, the angular factor ensures that a cell with constant pixel intensity along its outline will have zero axial polarity, even if its shape is elongated. For comparison among different cells, the cellular polarity nematic $\widetilde{\boldsymbol{A}}$ can be normalized by the sum of all pixel nematic magnitudes. Also in this case, the magnitude of the normalized cell nematic varies between zero and one.

Cell-based and interface-based methods typically yield in most cases similar results. However note that for strongly elongated cells, the results can differ.

\section{[Box] Mathematical representations of vectorial and axial polarity}

In order to represent polarity orientation and magnitude in $2 \mathrm{D}$, we recommend using vectors for vectorial polarity, and nematics for axial polarity. A vector $\boldsymbol{p}$ can be represented by an arrow (Fig. 1A). It consists of two components $\boldsymbol{p}=\left(p_{x}, p_{y}\right)$, which correspond to its projections on $x$ and $y$ axes, respectively. Based on these components, the polarity direction $\phi$ and magnitude $|p|$ (i.e. vector length) can be computed as follows:

$$
\phi=\arctan 2\left(p_{y}, p_{x}\right) \text { and }|p|=\sqrt{p_{x}^{2}+p_{y}{ }^{2}} .
$$

Here, "arctan2" denotes the arcus tangens function with two arguments. Conversely, the vector components can be computed from direction and length using the formulas

$$
p_{x}=|p| \cos (\phi) \text { and } p_{y}=|p| \sin (\phi) \text {. }
$$

It is easier to visualize vectors using the representation by angle $\phi$ and norm $|p|$, while the representation by components should be used for arithmetics. To average $n$ vectors, one first adds them and then divides their sum by $n$. To add two vectors, $\boldsymbol{p}_{1}=\left(p_{1, x}, p_{1, y}\right)$ and $\boldsymbol{p}_{2}=$ $\left(p_{2, x}, p_{2, y}\right)$, their respective components have to be added separately: $\boldsymbol{p}_{1}+\boldsymbol{p}_{2}=\left(p_{1, x}+\right.$ 
$p_{2, x}, p_{1, y}+p_{2, y}$ ). Similarly, to multiply (or divide) a vector by a number $n$, each of its components, $p_{x}$ and $p_{y}$, has to be multiplied (divided) by this number $n$.

A nematic $\widetilde{A}$ can be represented by a bar (Fig. 1B) and is composed of two components, $\tilde{A}_{x x}$ and $\tilde{A}_{x y}$. Similar to a vector, these components can be computed from the polarity orientation angle $\psi$ (i.e. the angle of the bar) and the polarity magnitude $|\tilde{A}|$ (the length of the bar):

and vice versa

$$
\tilde{A}_{x x}=|\tilde{A}| \cos (2 \psi) \quad \text { and } \quad \tilde{A}_{x y}=|\tilde{A}| \sin (2 \psi)
$$

$$
\psi=\frac{1}{2} \arctan 2\left(\tilde{A}_{x y}, \tilde{A}_{x x}\right) \text { and }|\tilde{A}|=\sqrt{\tilde{A}_{x x}{ }^{2}+\tilde{A}_{x y}{ }^{2}} .
$$

The representation by angle and magnitude is used to visualize nematics, whereas the representation by components is used for arithmetics, which works as for vectors.

Note that a bar representing a nematic is the same when you rotate it by 180 degrees, whereas a vector has to be rotated by 360 degrees to be the same. Consequently, while vectors add up to zero whenever they have the same lengths and opposing directions, two nematics add up to zero whenever they have the same magnitude and are perpendicular to each other. As a related example, two vectors of the same length add up to a vector with double length whenever they have the same direction (+/-360 degrees), while for nematics this is true whenever their axes are the same (+/-180 degrees).

\section{[End of box]}

\section{[[[ Desired position of Figure 3: somewhere within the section "Tissue deformation"]]]}

\section{B. Tissue deformation}

\section{Without segmentation}

Tissue deformation can be computed from the flow field extracted by PIV (see section IV.C). This method is fast and does not require segmentation, but does not provide information on the underlying cellular processes.

The underlying idea is that tissue deformation is induced by the relative motion of adjacent tissue regions, while a global translation does not affect tissue shape at all (Fig. 3B-D). For example, Fig. $3 \mathrm{C}$ shows an expansion of a tissue along the horizontal $(x)$ axis. This deformation corresponds to a velocity field whose $x$ component, $v_{x}$, increases towards the right. The corresponding rate of expansion can be measured by computing the relative increase of the velocity component $v_{x}$ per length unit in $x$ direction. Fig. 3D shows another example, where the velocity component $v_{x}$ is constant along $x$ direction, but increases in $y$ direction. This corresponds to a so-called "simple shear deformation". The rate of simple shear is the relative increase of the velocity component $v_{x}$ per length unit in $y$ direction. In addition to these two kinds of deformations, also the $y$ component of the velocity, $v_{y}$, may change in both $x$ and $y$ directions. The mathematical object that contains all four of the corresponding deformation 
rates is called "velocity gradient" and is used to describe local tissue deformation (see deformation box).

Deformations can be decomposed into a so-called "isotropic deformation component" and an "anisotropic deformation component" (Fig. 3E,F). This can be useful because certain cellular events, for instance T1 transitions, only contribute to one of the two, i.e. anisotropic deformation. The rates of both components can be extracted from the velocity gradient (see deformation box for details). Isotropic deformation refers to tissue expansion (or shrinkage) in all directions with the same rate, and is characterized by the isotropic deformation rate $V$.

Anisotropic deformation, also called "pure shear", refers to a convergence-extension-like tissue deformation, where the area of the tissue remains constant (Fig. 3F). While isotropic deformation is described by the single parameter $V$, pure shear deformation is characterized by the rate and the axis of tissue expansion. It is thus characterized by a nematic $\widetilde{V}$, like axial polarity (see polarity box). In addition to isotropic and anisotropic deformation rates, the rotation rate $\omega$ of a piece of tissue can also be computed from the velocity gradient (Fig. 3G, see deformation box).

Note that any deformation can be described as a combination of the four deformation modes shown in Figs. 3E-G. For instance, expansion along the $x$ axis is a combination of isotropic deformation and pure shear along the $x$ axis (Fig. $3 \mathrm{H}$ ). Moreover, simple shear is the combination of pure shear along a diagonal axis and rotation (Fig. 3I). Finally, beware that tissue deformations are typically spatially heterogeneous. The deformation rates $V$ and $\widetilde{\boldsymbol{V}}$ and the rotation rate $\omega$ provide local information about tissue deformation.

\section{[Box] Velocity gradient and tissue deformation}

The velocity gradient is a combination of the respective gradients of both velocity components, $v_{x}$ and $v_{y}$. For instance the gradient of $v_{x}$ can be understood like a morphogen gradient: it has a direction and a magnitude (its steepness). Thus, it can be represented by a vector with two components $\partial v_{x} / \partial x$ and $\partial v_{x} / \partial y$, which are the respective derivatives (i.e. changes) of $v_{x}$ in $x$ and $y$ direction. Hence, including $v_{y}$, the whole velocity gradient is composed of four different derivatives. These derivatives can be combined to compute the rates of isotropic deformation $V$, anisotropic deformation $\widetilde{\boldsymbol{V}}$ (which is a nematic, see polarity box), and rotation $\omega$ :

$$
V=\frac{\partial v_{x}}{\partial x}+\frac{\partial v_{y}}{\partial y}, \quad \tilde{V}_{x x}=\frac{1}{2}\left(\frac{\partial v_{x}}{\partial x}-\frac{\partial v_{y}}{\partial y}\right), \quad \tilde{V}_{x y}=\frac{1}{2}\left(\frac{\partial v_{y}}{\partial x}+\frac{\partial v_{x}}{\partial y}\right), \quad \omega=\frac{1}{2}\left(\frac{\partial v_{y}}{\partial x}-\frac{\partial v_{x}}{\partial y}\right) .
$$

With these definitions, the average rate of isotropic deformation $V$ corresponds to the rate of relative area expansion (or shrinkage for negative $V$ ): $V=\dot{A}_{T} / A_{T}$, where $A_{T}$ is the area of the tissue and the dot denotes the time derivative. Anisotropic deformation changes the aspect ratio of a tissue. In particular, if both anisotropic deformation and tissue elongation are oriented along the $x$ axis, the horizontal component of the anisotropic deformation rate corresponds to the relative change of the tissue aspect ratio $R: \widetilde{V}_{x x}=\dot{R} /(2 R)$.

\section{[End of box]}




\section{With segmentation}

PIV can be used to characterize tissue deformations, but not the underlying cellular events. To measure the contributions of individual cellular processes to tissue deformation, time-lapse movies need to be segmented and cells tracked over time (see section IV.D).

The contribution of individual cellular processes to both isotropic and anisotropic tissue deformation can be precisely quantified. Isotropic tissue deformation can be due to cell area changes, cell divisions, and cell extrusions. More precisely, the rate of isotropic tissue deformation can be decomposed into the relative change of average cell area $\underline{a}$ plus the normalized rate of cell divisions $k_{d}$ (number of divisions per cell per time unit) minus the normalized rate of cell extrusions $k_{e}$ (number of extrusions per cell per time unit) (Etournay et al., 2015; Matthias Merkel, 2014):

$$
V=\frac{1}{\bar{a}} \dot{\bar{a}}+k_{d}-k_{e} \text {. }
$$

This remains true also when divisions occur without cell area growth.

Decomposing the anisotropic part of tissue deformation into cellular contributions is more complex and several methods have been used (Blanchard et al., 2009; Brodland et al., 2006; Butler et al., 2009; Economou, Brock, Cobourne, \& Green, 2013; Etournay et al., 2015; Graner et al., 2008; Matthias Merkel, 2014; Rauzi et al., 2008). For instance, Blanchard and colleagues (Blanchard et al., 2009) quantify cell elongation by fitting ellipses to cell shapes. Note that throughout this section, "elongation" refers to shape anisotropy, not the process of elongating. Tissue deformation is obtained for small patches of cells by linearly fitting the movement of all cell centers within a given patch. The contribution of cell intercalation is quantified for each patch by subtracting the change of average cell elongation from the total patch deformation.

Another method by Graner and colleagues (Graner et al., 2008; Guirao et al., 2015) describes the elongation of a cell using the connection lines between its center and the centers of all neighboring cells. Tissue deformation induces continuous changes in these connection lines. In contrast, T1 transitions, cell divisions, and cell extrusions induce rewiring of the connections. By comparing the connection lines before and after the respective topological transition, the contribution of the transition to tissue deformation can be extracted.

In the following, we focus on the so-called "Triangle Method" (Etournay et al., 2015; Matthias Merkel, 2014). First, the centers of neighboring cells are connected by triangles (Fig. 3J). The elongation of each triangle is quantified by a nematic $\widetilde{\mathbf{q}}$ (Fig. 3K), which can be used as proxy to define cell elongation (Fig. 3L). Upon cell motion and deformation, each triangle deforms with an anisotropic deformation rate $\tilde{v}$ (i.e. pure shear rate, Fig. 3M), which corresponds to the change of triangle elongation $\widetilde{\mathbf{q}}$. The average triangle pure shear rate corresponds to the overall anisotropic tissue deformation rate $\widetilde{\boldsymbol{V}}$.

The triangles are used to define the respective contributions of the different cellular events to the tissue pure shear rate $\widetilde{\boldsymbol{V}}$ (Fig. 3A). In the simple case where all cells deform in the same way without topological transitions, the overall tissue pure shear rate $\widetilde{\boldsymbol{V}}$ corresponds to the change of the average triangle elongation, which we denote by $\widetilde{\boldsymbol{Q}}: \widetilde{\boldsymbol{V}}=\mathrm{D} \widetilde{\boldsymbol{Q}} / \mathrm{D} t$. An example is given in Fig. $3 \mathrm{~N}$, where four cells elongate horizontally with a constant shear rate $\widetilde{\boldsymbol{V}}$ (Fig. 30), reflected in the linear increase of the average triangle elongation $\widetilde{\boldsymbol{Q}}$ (Fig. 3P).

Fig. 3Q illustrates how the contribution of $\mathrm{T} 1$ transitions to the pure shear rate is quantified by the Triangle Method. Shown is a process with a constant shear rate along the 
horizontal axis with a T1 transition. As there is no net change in the cell elongation (i.e. the average triangle elongation) (Fig. 3Q), the $\mathrm{T} 1$ transition has to fully account for the deformation during this process. In particular, at the instant of the cell neighbor exchange, two triangles disappear and two new triangles appear (Fig. 3Q,S). Consequently, the average triangle elongation along the horizontal axis suddenly decreases (schemes and blue arrow in Figs. 3S). This decrease in triangle elongation quantifies the overall contribution of the T1 transition to pure shear. Note that also rosette formation and resolution can be accounted for in this way (details on how to handle vertices with more than three cells are provided in (Etournay et al., 2015; Matthias Merkel, 2014)).

To define contributions by cell divisions and $\mathrm{T} 2$ transitions to anisotropic deformation, the Triangle Method follows the same principle. In particular, during a cell division two triangles appear, whereas during a $\mathrm{T} 2$ transition, two triangles disappear.

Finally, the Triangle Method reveals an additional contribution to anisotropic tissue deformation, which is due to collective cell motion. In general, this contribution appears whenever triangle elongation and local tissue rotation or local area expansion vary across a tissue (see details in_(Etournay et al., 2015; Matthias Merkel, 2014)).

All three methods described here require segmentation and cell tracking. While the method by Blanchard et al. relies on cell outlines, the other two methods use only information about cell centers and cell neighborship. The first method represents cell shape more directly, but does not take into account cell divisions and cell extrusions, while the other two methods do. Finally, the Triangle Method is so far the only method that captures the contribution by collective cell motion.

A software suite that provides an implementation of the Triangle Method is available as part of the free tool "TissueMiner". This tool together with hands-on explanations and step-bystep tutorials can be found in (Etournay et al., 2016).

Finally, note that also Heemskerk and colleagues (Heemskerk et al., 2014) use triangles to quantify tissue deformation. However there the triangles are much larger; triangle corners are defined by the centers of clones. This method is thus suited to quantify large-scale tissue deformation. In contrast, since the Triangle Method defines the triangles at the cellular scale, the deformation of each individual cellular event can be precisely measured.

\section{Averaging of tissue deformation and polarity patterns}

Local averaging of deformation and cell- or clone-based polarity can be used to reveal their large-scale patterns and improve S/N ratio (Aigouy et al., 2010; Guirao et al., 2015; M. Merkel et al., 2014; Sagner et al., 2012). This can be done by dividing the image into blocks and by averaging measured quantities within each block. Note that in order to average oriented objects like vectors or nematics, their components need to be averaged separately. In particular, angles should never be averaged. Such blocks should be sufficiently large to ensure a good S/N ratio, but also small enough to preserve local differences between neighboring regions of the tissue. Note that averaging over several samples requires their alignment in space and time using tissue-specific landmarks (e.g. morphological landmarks, characteristic cell and tissue behaviors, etc.; (Aigouy et al., 2010; Collinet et al., 2015; Guirao et al., 2015; M. Merkel et al., 2014; Sagner et al., 2012)). 


\section{Concluding remarks}

Cell deformations, divisions, rearrangements and extrusions are well known to participate in epithelial morphogenesis. In many cases these events are coordinated within an epithelium through the action of planar polarized proteins. However, the extent to which each of these events contributes to overall tissue reshaping is not always obvious. In particular, the fact that a cellular event is frequent does not necessarily imply that it significantly contributes to morphogenesis. This highlights the importance of the development of new approaches to quantitatively study epithelial tissue deformations and planar polarity from the cell to tissue scale. In this review, we outline biological tools, imaging approaches and computational methods to quantify the individual contributions of cellular events to morphogenesis. These enhance our understanding of epithelial morphogenesis by allowing direct summation over all the individual events that occur at the cellular level, and even linking them to molecular mechanisms. Finally, such precise quantifications allow to refine and test quantitative models that are expected to broaden our understanding of this fundamental biological process.

\section{Acknowledgement}

We thank members of the Lecuit lab, for discussions etc. C.C. is supported by a Human Frontiers Science Program Long-Term Fellowship and by a postdoctoral fellowship of the Fondation Recherche Medicale. M.M. is supported by the Alfred P. Sloan Foundation, the Gordon and Betty Moore Foundation and NSF-DMR-1352184. A.S. is supported by a Human Frontiers Science Program Long-Term Fellowship, Marie Curie Intra-European Fellowship and EMBO LongTerm Fellowship.

\section{References}

Aigouy, B., Farhadifar, R., Staple, D. B., Sagner, A., Roper, J. C., Julicher, F., \& Eaton, S. (2010). Cell flow reorients the axis of planar polarity in the wing epithelium of Drosophila. Cell, 142(5), 773-786. doi:10.1016/j.cell.2010.07.042

Ambegaonkar, A. A., \& Irvine, K. D. (2015). Coordination of planar cell polarity pathways through Spiny legs. elife, 4, e09946. doi:10.7554/eLife.09946

Baena-Lopez, L. A., Baonza, A., \& Garcia-Bellido, A. (2005). The orientation of cell divisions determines the shape of Drosophila organs. Curr Biol, 15(18), 1640-1644. doi:10.1016/j.cub.2005.07.062

Bertet, C., Sulak, L., \& Lecuit, T. (2004). Myosin-dependent junction remodelling controls planar cell intercalation and axis elongation. Nature, 429(6992), 667-671. doi:10.1038/nature02590

Besson, C., Bernard, F., Corson, F., Rouault, H., Reynaud, E., Keder, A., . . Schweisguth, F. (2015). Planar Cell Polarity Breaks the Symmetry of PAR Protein Distribution prior to Mitosis in Drosophila Sensory Organ Precursor Cells. Curr Biol, 25(8), 1104-1110. doi:10.1016/j.cub.2015.02.073

Blanchard, G. B., Kabla, A. J., Schultz, N. L., Butler, L. C., Sanson, B., Gorfinkiel, N., . . Adams, R. J. (2009). Tissue tectonics: morphogenetic strain rates, cell shape change and intercalation. Nature Methods, 6, 458-464. doi:10.1038/nmeth.1327 
Blankenship, J. T., Backovic, S. T., Sanny, J. S., Weitz, O., \& Zallen, J. A. (2006). Multicellular rosette formation links planar cell polarity to tissue morphogenesis. Dev Cell, 11(4), 459470. doi:10.1016/j.devcel.2006.09.007

Bohn, S. (2003). Bubbles under stress. Eur Phys J E Soft Matter, 11(2), 177-189. doi:10.1140/epje/i2003-10014-x

Bosveld, F., Bonnet, I., Guirao, B., Tlili, S., Wang, Z., Petitalot, A., . . . Bellaiche, Y. (2012). Mechanical Control of Morphogenesis by Fat/Dachsous/Four-Jointed Planar Cell Polarity Pathway. Science, 336, 724-727. doi:10.1126/science.1221071

Bosveld, F., Markova, O., Guirao, B., Martin, C., Wang, Z., Pierre, A., . . Bellaïche, Y. (2016). Epithelial tricellular junctions act as interphase cell shape sensors to orient mitosis. Nature. doi:10.1038/nature16970

Brittle, A., Thomas, C., \& Strutt, D. (2012). Planar polarity specification through asymmetric subcellular localization of fat and dachsous. Current Biology, 22, 907-914. doi:10.1016/j.cub.2012.03.053

Brodland, G. W., Chen, D. I. L., \& Veldhuis, J. H. (2006). A cell-based constitutive model for embryonic epithelia and other planar aggregates of biological cells. International Journal of Plasticity, 22, 965-995. doi:10.1016/j.ijplas.2005.05.002

Butler, L. C., Blanchard, G. B., Kabla, A. J., Lawrence, N. J., Welchman, D. P., Mahadevan, L., . . Sanson, B. (2009). Cell shape changes indicate a role for extrinsic tensile forces in Drosophila germ-band extension. Nature Cell Biology, 11, 859-864. doi:10.1038/ncb1894

Campinho, P., Behrndt, M., Ranft, J., Risler, T., Minc, N., \& Heisenberg, C. P. (2013). Tensionoriented cell divisions limit anisotropic tissue tension in epithelial spreading during zebrafish epiboly. Nat Cell Biol, 15(12), 1405-1414. doi:10.1038/ncb2869

Chacon-Heszele, M. F., Ren, D., Reynolds, A. B., Chi, F., \& Chen, P. (2012). Regulation of cochlear convergent extension by the vertebrate planar cell polarity pathway is dependent on p120-catenin. Development, 139(5), 968-978. doi:10.1242/dev.065326

Chae, J., Kim, M. J., Goo, J. H., Collier, S., Gubb, D., Charlton, J., . . . Park, W. J. (1999). The Drosophila tissue polarity gene starry night encodes a member of the protocadherin family. Development, 126(23), 5421-5429.

Cilla, R., Mechery, V., Hernandez de Madrid, B., Del Signore, S., Dotu, I., \& Hatini, V. (2015). Segmentation and tracking of adherens junctions in 3D for the analysis of epithelial tissue morphogenesis. PLoS Comput Biol, 11(4), e1004124. doi:10.1371/journal.pcbi.1004124

Collinet, C., Rauzi, M., Lenne, P. F., \& Lecuit, T. (2015). Local and tissue-scale forces drive oriented junction growth during tissue extension. Nat Cell Biol, 17(10), 1247-1258. doi:10.1038/ncb3226

Danielian, P. S., Muccino, D., Rowitch, D. H., Michael, S. K., \& McMahon, A. P. (1998). Modification of gene activity in mouse embryos in utero by a tamoxifen-inducible form of Cre recombinase. Curr Biol, 8(24), 1323-1326.

de Medeiros, G., Norlin, N., Gunther, S., Albert, M., Panavaite, L., Fiuza, U. M., . . Hufnagel, L. (2015). Confocal multiview light-sheet microscopy. Nat Commun, 6, 8881. doi:10.1038/ncomms9881

Denk, W., Strickler, J. H., \& Webb, W. W. (1990). Two-photon laser scanning fluorescence microscopy. Science, 248(4951), 73-76.

Denk, W., \& Svoboda, K. (1997). Photon upmanship: why multiphoton imaging is more than a gimmick. Neuron, 18(3), 351-357.

Devenport, D., Oristian, D., Heller, E., \& Fuchs, E. (2011). Mitotic internalization of planar cell polarity proteins preserves tissue polarity. Nature Cell Biology, 13, 893-902. doi:10.1038/ncb2284 
Economou, A. D., Brock, L. J., Cobourne, M. T., \& Green, J. B. a. (2013). Whole population cell analysis of a landmark-rich mammalian epithelium reveals multiple elongation mechanisms. Development, 140, 4740-4750. doi:10.1242/dev.096545

Eisenhoffer, G. T., Loftus, P. D., Yoshigi, M., Otsuna, H., Chien, C. B., Morcos, P. A., \& Rosenblatt, J. (2012). Crowding induces live cell extrusion to maintain homeostatic cell numbers in epithelia. Nature, 484(7395), 546-549. doi:10.1038/nature10999

Eisenhoffer, G. T., \& Rosenblatt, J. (2013). Bringing balance by force: live cell extrusion controls epithelial cell numbers. Trends Cell Biol, 23(4), 185-192. doi:10.1016/j.tcb.2012.11.006

Etournay, R., Merkel, M., Popović, M., Brandl, H., Dye, N. A., Aigouy, B., . . Jülicher, F. (2016). TissueMiner: a multiscale analysis toolkit to quantify how cellular processes create tissue dynamics. elife, 5. doi:10.7554/eLife.14334

Etournay, R., Popović, M., Merkel, M., Nandi, A., Blasse, C., Aigouy, B., . . . Eaton, S. (2015). Interplay of cell dynamics and epithelial tension during morphogenesis of the Drosophila pupal wing. elife, 4, e07090. doi:10.7554/eLife.07090

Forster, B., Van De Ville, D., Berent, J., Sage, D., \& Unser, M. (2004). Complex wavelets for extended depth-of-field: a new method for the fusion of multichannel microscopy images. Microsc Res Tech, 65(1-2), 33-42. doi:10.1002/jemt.20092

Ganguly, S., Williams, L. S., Palacios, I. M., \& Goldstein, R. E. (2012). Cytoplasmic streaming in Drosophila oocytes varies with kinesin activity and correlates with the microtubule cytoskeleton architecture. Proc Natl Acad Sci U S A, 109(38), 15109-15114. doi:10.1073/pnas.1203575109

Gelbart, M. A., He, B., Martin, A. C., Thiberge, S. Y., Wieschaus, E. F., \& Kaschube, M. (2012). Volume conservation principle involved in cell lengthening and nucleus movement during tissue morphogenesis. Proc Natl Acad Sci U S A, 109(47), 19298-19303. doi:10.1073/pnas.1205258109

Golic, K. G. (1991). Site-specific recombination between homologous chromosomes in Drosophila. Science, 252(5008), 958-961.

Goodrich, L. V., \& Strutt, D. (2011). Principles of planar polarity in animal development. Development, 138(10), 1877-1892. doi:10.1242/dev.054080

Graner, F., Dollet, B., Raufaste, C., \& Marmottant, P. (2008). Discrete rearranging disordered patterns, part I: Robust statistical tools in two or three dimensions. The European Physical Journal E, 25, 349-369. doi:10.1140/epje/i2007-10298-8

Guillot, C., \& Lecuit, T. (2013). Mechanics of epithelial tissue homeostasis and morphogenesis. Science, 340(6137), 1185-1189. doi:10.1126/science.1235249

Guirao, B., Rigaud, S. U., Bosveld, F., Bailles, A., López-Gay, J., Ishihara, S., . . Bellaïche, Y. (2015). Unified quantitative characterization of epithelial tissue development. elife, 4. doi:10.7554/elife.08519

Hayashi, S., \& McMahon, A. P. (2002). Efficient recombination in diverse tissues by a tamoxifeninducible form of Cre: a tool for temporally regulated gene activation/inactivation in the mouse. Dev Biol, 244(2), 305-318. doi:10.1006/dbio.2002.0597

He, B., Doubrovinski, K., Polyakov, O., \& Wieschaus, E. (2014). Apical constriction drives tissuescale hydrodynamic flow to mediate cell elongation. Nature, 508(7496), 392-396. doi:10.1038/nature13070

Heemskerk, I., Lecuit, T., \& LeGoff, L. (2014). Dynamic clonal analysis based on chronic in vivo imaging allows multiscale quantification of growth in the Drosophila wing disc. Development, 141(11), 2339-2348. doi:10.1242/dev.109264

Heller, D., Hoppe, A., Restrepo, S., Gatti, L., Tournier, A. L., Tapon, N., . . Mao, Y. (2016). EpiTools: An Open-Source Image Analysis Toolkit for Quantifying Epithelial Growth Dynamics. Dev Cell, 36(1), 103-116. doi:10.1016/j.devcel.2015.12.012

Helmchen, F., \& Denk, W. (2005). Deep tissue two-photon microscopy. Nat Methods, 2(12), 932940. doi:10.1038/nmeth 818 
Huisken, J., \& Stainier, D. Y. (2007). Even fluorescence excitation by multidirectional selective plane illumination microscopy (mSPIM). Opt Lett, 32(17), 2608-2610.

Huisken, J., \& Stainier, D. Y. (2009). Selective plane illumination microscopy techniques in developmental biology. Development, 136(12), 1963-1975. doi:10.1242/dev.022426

Irvine, K. D., \& Wieschaus, E. (1994). Cell intercalation during Drosophila germband extension and its regulation by pair-rule segmentation genes. Development, 120(4), 827-841.

Karner, C. M., Chirumamilla, R., Aoki, S., Igarashi, P., Wallingford, J. B., \& Carroll, T. J. (2009). Wnt9b signaling regulates planar cell polarity and kidney tubule morphogenesis. Nat Genet, 41(7), 793-799. doi:10.1038/ng.400

Keller, P. J., \& Stelzer, E. H. (2008). Quantitative in vivo imaging of entire embryos with Digital Scanned Laser Light Sheet Fluorescence Microscopy. Curr Opin Neurobiol, 18(6), 624632. doi:10.1016/j.conb.2009.03.008

Khan, Z., Wang, Y. C., Wieschaus, E. F., \& Kaschube, M. (2014). Quantitative 4D analyses of epithelial folding during Drosophila gastrulation. Development, 141(14), 2895-2900. doi:10.1242/dev.107730

Kretzschmar, K., \& Watt, F. M. (2012). Lineage tracing. Cell, 148(1-2), 33-45. doi:10.1016/j.cell.2012.01.002

Krzic, U., Gunther, S., Saunders, T. E., Streichan, S. J., \& Hufnagel, L. (2012). Multiview light-sheet microscope for rapid in toto imaging. Nat Methods, 9(7), 730-733. doi:10.1038/nmeth.2064

Legoff, L., Rouault, H., \& Lecuit, T. (2013). A global pattern of mechanical stress polarizes cell divisions and cell shape in the growing Drosophila wing disc. Development, 140(19), 4051-4059. doi:10.1242/dev.090878

Leung, C. Y., \& Fernandez-Gonzalez, R. (2015). Quantitative image analysis of cell behavior and molecular dynamics during tissue morphogenesis. Methods Mol Biol, 1189, 99-113. doi:10.1007/978-1-4939-1164-6_7

Levayer, R., \& Lecuit, T. (2013). Oscillation and polarity of E-cadherin asymmetries control actomyosin flow patterns during morphogenesis. Dev Cell, 26(2), 162-175. doi:10.1016/j.devcel.2013.06.020

Levayer, R., Pelissier-Monier, A., \& Lecuit, T. (2011). Spatial regulation of Dia and Myosin-II by RhOGEF2 controls initiation of E-cadherin endocytosis during epithelial morphogenesis. Nat Cell Biol, 13(5), 529-540. doi:10.1038/ncb2224

Lienkamp, S. S., Liu, K., Karner, C. M., Carroll, T. J., Ronneberger, O., Wallingford, J. B., \& Walz, G. (2012). Vertebrate kidney tubules elongate using a planar cell polarity-dependent, rosette-based mechanism of convergent extension. Nat Genet, 44(12), 1382-1387. doi:10.1038/ng.2452

Lynch, H. E., Veldhuis, J., Brodland, G. W., \& Hutson, M. S. (2014). Modeling cell elongation during germ band retraction: cell autonomy versus applied anisotropic stress. New J Phys, 16(2014), 055003. doi:10.1088/1367-2630/16/5/055003

Marcinkevicius, E., Fernandez-Gonzalez, R., \& Zallen, J. A. (2009). Q\&A: quantitative approaches to planar polarity and tissue organization. J Biol, 8(12), 103. doi:10.1186/jbiol191

Marinari, E., Mehonic, A., Curran, S., Gale, J., Duke, T., \& Baum, B. (2012). Live-cell delamination counterbalances epithelial growth to limit tissue overcrowding. Nature, 484(7395), 542545. doi:10.1038/nature10984

Matis, M., Axelrod, J. D., \& Galic, M. (2012). A Universal Analysis Tool for the Detection of Asymmetric Signal Distribution in Microscopic Images. 1301-1309. doi:10.1002/dvdy.23818

Matis, M., Russler-Germain, D. a., Hu, Q., Tomlin, C. J., \& Axelrod, J. D. (2014). Microtubules provide directional information for core PCP function. elife, 3, e02893. doi:10.7554/eLife.02893 
Mayer, M., Depken, M., Bois, J. S., Julicher, F., \& Grill, S. W. (2010). Anisotropies in cortical tension reveal the physical basis of polarizing cortical flows. Nature, 467(7315), 617-621. doi:10.1038/nature09376

Merkel, M. (2014). From cells to tissues: Remodeling and polarity reorientation in epithelial tissues.

Merkel, M., Sagner, A., Gruber, F. S., Etournay, R., Blasse, C., Myers, E., . . Julicher, F. (2014). The balance of prickle/spiny-legs isoforms controls the amount of coupling between core and fat PCP systems. Curr Biol, 24(18), 2111-2123. doi:10.1016/j.cub.2014.08.005

Mosaliganti, K. R., Noche, R. R., Xiong, F., Swinburne, I. A., \& Megason, S. G. (2012). ACME: automated cell morphology extractor for comprehensive reconstruction of cell membranes. PLoS Comput Biol, 8(12), e1002780. doi:10.1371/journal.pcbi.1002780

Munjal, A., Philippe, J. M., Munro, E., \& Lecuit, T. (2015). A self-organized biomechanical network drives shape changes during tissue morphogenesis. Nature, 524(7565), 351355. doi:10.1038/nature14603

Nagy, A. (2000). Cre recombinase: the universal reagent for genome tailoring. Genesis, 26(2), 99109.

Nishimura, T., Honda, H., \& Takeichi, M. (2012). Planar cell polarity links axes of spatial dynamics in neural-tube closure. Cell, 149(5), 1084-1097. doi:10.1016/j.cell.2012.04.021

Nishimura, T., \& Takeichi, M. (2008). Shroom3-mediated recruitment of Rho kinases to the apical cell junctions regulates epithelial and neuroepithelial planar remodeling. Development, 135(8), 1493-1502. doi:10.1242/dev.019646

Pal, C., Chakrabarti, A., \& Ghosh, R. (2015). A Brief Survey of Recent Edge-Preserving Smoothing Algorithms on Digital Images. Procedia Computer Science, ArXiv e-prints.

Preibisch, S., Saalfeld, S., \& Tomancak, P. (2009). Globally optimal stitching of tiled 3D microscopic image acquisitions. Bioinformatics, 25(11), 1463-1465. doi:10.1093/bioinformatics/btp184

Raffel, M. (2007). Particle image velocimetry : a practical guide (2nd ed.). Heidelberg ; New York: Springer.

Rauzi, M., Verant, P., Lecuit, T., \& Lenne, P.-F. (2008). Nature and anisotropy of cortical forces orienting Drosophila tissue morphogenesis. Nature Cell Biology, 10, 1401-1410. doi:10.1038/ncb1798

Rezakhaniha, R., Agianniotis, A., Schrauwen, J. T. C., Griffa, A., Sage, D., Bouten, C. V. C., . . Stergiopulos, N. (2012). Experimental investigation of collagen waviness and orientation in the arterial adventitia using confocal laser scanning microscopy. Biomechanics and Modeling in Mechanobiology, 11, 461-473. doi:10.1007/s10237-011-0325-z

Sagner, A., Merkel, M., Aigouy, B., Gaebel, J., Brankatschk, M., Jülicher, F., \& Eaton, S. (2012). Establishment of Global Patterns of Planar Polarity during Growth of the Drosophila Wing Epithelium. Current Biology, 22, 1296-1301. doi:10.1016/j.cub.2012.04.066

Sauer, B., \& Henderson, N. (1988). Site-specific DNA recombination in mammalian cells by the Cre recombinase of bacteriophage P1. Proc Natl Acad Sci U S A, 85(14), 5166-5170.

Schindelin, J., Arganda-Carreras, I., Frise, E., Kaynig, V., Longair, M., Pietzsch, T., . . Cardona, A. (2012). Fiji: an open-source platform for biological-image analysis. Nat Methods, 9(7), 676-682. doi:10.1038/nmeth.2019

Schneider, C. A., Rasband, W. S., \& Eliceiri, K. W. (2012). NIH Image to ImageJ: 25 years of image analysis. Nat Methods, 9(7), 671-675.

Simoes Sde, M., Blankenship, J. T., Weitz, O., Farrell, D. L., Tamada, M., Fernandez-Gonzalez, R., \& Zallen, J. A. (2010). Rho-kinase directs Bazooka/Par-3 planar polarity during Drosophila axis elongation. Dev Cell, 19(3), 377-388. doi:10.1016/j.devcel.2010.08.011

So, P. T., Dong, C. Y., Masters, B. R., \& Berland, K. M. (2000). Two-photon excitation fluorescence microscopy. Annu Rev Biomed Eng, 2, 399-429. doi:10.1146/annurev.bioeng.2.1.399 
Srinivas, S., Watanabe, T., Lin, C. S., William, C. M., Tanabe, Y., Jessell, T. M., \& Costantini, F. (2001). Cre reporter strains produced by targeted insertion of EYFP and ECFP into the ROSA26 locus. BMC Dev Biol, 1, 4.

Stojmenovic, M., \& Nayak, A. (2007). Direct ellipse fitting and measuring based on shape boundaries. Advances in Image and Video Technology, Proceedings, 4872, 221-235.

Supatto, W., Debarre, D., Moulia, B., Brouzes, E., Martin, J. L., Farge, E., \& Beaurepaire, E. (2005). In vivo modulation of morphogenetic movements in Drosophila embryos with femtosecond laser pulses. Proc Natl Acad Sci U S A, 102(4), 1047-1052. doi:10.1073/pnas.0405316102

Tada, M., \& Kai, M. (2012). Planar cell polarity in coordinated and directed movements. Curr Top Dev Biol, 101, 77-110. doi:10.1016/B978-0-12-394592-1.00004-1

Tamada, M., Farrell, D. L., \& Zallen, J. A. (2012). Abl regulates planar polarized junctional dynamics through beta-catenin tyrosine phosphorylation. Dev Cell, 22(2), 309-319. doi:10.1016/j.devcel.2011.12.025

Usui, T., Shima, Y., Shimada, Y., Hirano, S., Burgess, R. W., Schwarz, T. L., . . Uemura, T. (1999). Flamingo, a seven-pass transmembrane cadherin, regulates planar cell polarity under the control of Frizzled. Cell, 98(5), 585-595. doi:S0092-8674(00)80046-X [pii]

Vincent, L., \& Soille, P. (1991). Watersheds in Digital Spaces - an Efficient Algorithm Based on Immersion Simulations. leee Transactions on Pattern Analysis and Machine Intelligence, 13(6), 583-598. doi:Doi 10.1109/34.87344

Walck-Shannon, E., \& Hardin, J. (2014). Cell intercalation from top to bottom. Nat Rev Mol Cell Biol, 15(1), 34-48. doi:10.1038/nrm3723

Wallingford, J. B. (2012). Planar cell polarity and the developmental control of cell behavior in vertebrate embryos. Annu Rev Cell Dev Biol, 28, 627-653. doi:10.1146/annurev-cellbio092910-154208

Wang, J., Mark, S., Zhang, X., Qian, D., Yoo, S. J., Radde-Gallwitz, K., . . Chen, P. (2005). Regulation of polarized extension and planar cell polarity in the cochlea by the vertebrate PCP pathway. Nat Genet, 37(9), 980-985. doi:10.1038/ng1622

Weaire, D. L., \& Hutzler, S. (2001). The Physics of Foams. 246.

Weber, M., \& Huisken, J. (2011). Light sheet microscopy for real-time developmental biology. Curr Opin Genet Dev, 21(5), 566-572. doi:10.1016/j.gde.2011.09.009

$\mathrm{Xu}, \mathrm{T}$., \& Rubin, G. M. (1993). Analysis of genetic mosaics in developing and adult Drosophila tissues. Development, 117(4), 1223-1237.

Zallen, J. A., \& Wieschaus, E. (2004). Patterned gene expression directs bipolar planar polarity in Drosophila. Dev Cell, 6(3), 343-355. 


\section{Figure legends}

\section{Figure 1 Axial and vectorial polarities in cells and tissues.}

(A) Three examples showing an asymmetric distribution of a membrane-associated polarity protein (red) within a cell. This kind of polarity is visually represented by an arrow and can be quantified by a vector. Throughout the figure, cell membrane is shown in gray.

(B) Two examples of axial polarity for a single cell. Axial polarity is characterized by the presence of same amounts of a polarity protein on opposing sides of a cell. Magnitude and orientation of axial polarity is represented by a bar and can be quantified by a "nematic".

(C) In the absence of protein asymmetries, neither vectorial nor axial polarity can be detected (yellow dot).

(D-M) Tissues with axial and vectorial polarities and how they appear in microscopy images (F$H, K-M)$. (D) Tissue composed of axially polarized cells (left cell in (B)). (E) Tissue composed of vectorially polarized cells (middle cell in (A)). (F) Vectorial and axial polarity appear axial in microscopy images. $(\mathrm{G}, \mathrm{H})$ Mosaic tissue containing cells expressing a tagged PCP protein (green dot) surrounded by unlabeled cells. Mosaics allow to discriminate between axial (G) and vectorial $(H)$ polarities. Also, the direction of vectorial polarity is made evident $(H)$.

(I) Tissue composed of vectorially polarized cells (right cell in (A)). (J) Tissue composed of nonpolarized cells (left cell in (C)). (K) Vectorially polarized and nonpolarized cells both appear unpolarized in microscopy images. (L,M) Mosaics can discriminate between vectorially polarized cells (L) and nonpolarized cells (M).

$\left(\mathrm{N}-\mathrm{S}^{\prime}\right)$ Axial and vectorial polarity measurements using mosaics. $(\mathrm{N})$

(O) PCP staining in the pupal wing of Drosophila. Note that the cells exhibit an axial polarity

(P) Axial cell polarity is quantified by a nematic, which is the sum of individual pixel nematics (green bar) that pass through the cell center (green circle) and the respective pixel (magenta). The magnitude of each pixel nematic is given by the pixel intensity times the angular distance of neighboring pixels (red).

(Q) Mosaic tissue containing cells expressing tagged PCP proteins (green dots). (R) Isolated cell expressing a tagged polarity protein surrounded by unlabeled cells. $\left(R^{\prime}\right)$ Corresponding vectorial polarity measurement for an isolated cell, as described in (Besson et al., 2015). The cell polarity vector is the sum of polarity vectors for the individual pixels of the cell outline (green arrows), which point from the cell center (green dot) to the respective pixel. The length of each pixel vector is the pixel intensity times the distance between cell center and pixel. (S) Clonal population composed of three cells expressing a tagged PCP protein, surrounded by unlabeled cells. $\left(S^{\prime}\right)$ Corresponding vectorial polarity measurement for this 3-cell clone [Sagner 2012]. The polarity vector of the clone (yellow) is the sum of polarity vectors (green arrows) computed for all interfaces (blue lines) that outline the clone. The vector for a given interface is perpendicular to the interface pointing outside the clone, and the vector length corresponds to the sum of pixel intensities along the segmented interface. 


\section{Figure 2 Projection algorithms and cell segmentation.}

(A) (Top) Image of an epithelial tissue. (Middle) Blow up of the same image, note that an image is composed of picture elements (pixels). (Bottom) Images are nothing but a matrix of pixel intensities (red numbers).

(B) Examples of pixel-based 2D projection algorithms. (Left) Series of two-dimensional $(x, y)$ optical sections (along the $z$ axis) of an epithelium. (Middle) Max projection of the pixels along the dashed green line. The Max projection algorithm finds the pixel with the maximum intensity along the $z$ axis (asterisk). This pixel is used in the 2D projection (arrow). (Right) Average (Avg) projection of the pixels along the dashed green line. The average pixel intensity is used in the 2D projection.

(C) Example of a block-based 2D projection algorithm. The stack is cut into a series of blocks. Each block spans the whole $z$ axis and has defined width and height. One such block, located on the top left side of the stack, is indicated. In most block-based projections, only the most informative $z$ plane of the block is kept for the 2D projection (not shown).

(D) Results of the extended depth of field (left), maximum (middle) and average (right) 2D projections of the stack shown in (B).

(D') Result of the Stack Focuser projection (left). Corresponding stack focuser height map indicating the depth of the $z$ plane used for 2D projection. White blocks originate from deeper $z$ planes than blue ones.

(E) Watershed segmentation of the cell shown in (A). Each basin, i.e. cell cytoplasm, is attributed a unique color. Cell outlines are indicated by white pixels.

(F) Pixels of the perimeter of the central cell in (E) are outlined (left). Perimeter length is quantified as the sum of the lengths of the (yellow) lines connecting the centers of adjacent pixels (red squares). (Right) Blow up of the region indicated by a rectangle on the left image. A vertical or horizontal connection is 1 pixel long and an oblique one line has a length equal to $\sqrt{2}$, i.e. the diagonal of a pixel.

(G) (Left) Blow up of the region indicated by a rectangle in (E). (Middle) blow ups of two $3 \times 3$ squares located at two positions along the outline of the dark blue cell ( 1 and 2). Counting the different basin colors surrounding the central pixel (indicated by a yellow circle) of a square allows to determine if a pixel belongs to a boundary (i.e. is surrounded by exactly two different basins) or to a vertex (i.e. is surrounded by 3 or more basins). (Right) Image with segmented vertices indicated in red, cell boundary pixels labeled with random colors and cytoplasmic pixels indicated in black.

\section{Figure 3 Quantification of large-scale tissue deformation and} cell-scale contributions to it.

(A) Cellular processes underlying tissue morphogenesis.

(B) A uniform translation does not deform the tissue.

(C,D) Tissue deformation is created by spatial variation of the velocity. (C) A (uniaxial) expansion is created by increasing the horizontal component of the velocity along the $x$ axis. (D) A simple shear deformation is created by increasing the horizontal component of the velocity along the $y$ axis. 
(E-G) Based on the velocity gradient, the rates of (E) isotropic deformation, $V$, (F) anisotropic deformation, $\widetilde{\boldsymbol{V}}$ and $(\mathrm{G})$ rotation, $\omega$, can be computed.

(H) Uniaxial expansion can be decomposed into an isotropic and an anisotropic part.

(I) Simple shear deformation can be decomposed into an anisotropic deformation (i.e. pure shear) and a rotation.

(J-T) Triangle Method to quantify the contributions of individual cellular events to tissue deformation.

(J) A triangle (red) is created by connecting the respective centers (green) of three neighboring cells.

(K) The elongation (i.e. shape anisotropy) of a single triangle is quantified by the nematic $\widetilde{\boldsymbol{q}}$.

(L) The elongation of a cell (blue) is computed by averaging the elongations $\widetilde{\boldsymbol{q}}$ of the triangles (red) that have one of their corners at the center of this cell.

(M) The anisotropic deformation of a single triangle is quantified by the nematic $\widetilde{\boldsymbol{v}}$.

$(\mathrm{N}-\mathrm{P})$ Pure shear of four cells along the horizontal axis with a constant pure shear rate $(\mathrm{O})$ leads to a linear increase in the average triangle elongation (P).

(Q-S) Pure shear deformation with a constant rate (R) during which a T1 transition occurs. Because the net cell elongation does not change between beginning and end $(Q)$, the T1 transition accounts for the whole pure shear deformation. Note that the cell neighbor exchange removes and adds two triangles $(\mathrm{Q}, \mathrm{S})$. This induces an instantaneous reduction of the average triangle elongation along the horizontal axis at the instant of the neighbor exchange (schemes and blue arrow in S).

Note that panels $\mathrm{O}, \mathrm{P}, \mathrm{R}$, and $\mathrm{S}$ show the horizontal component of pure shear rate and average triangle elongation. 
$\mathbf{A}$
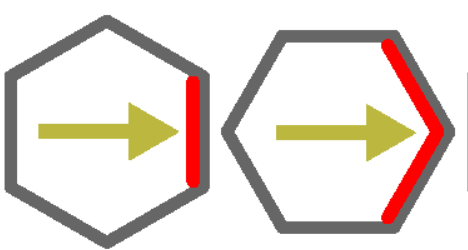
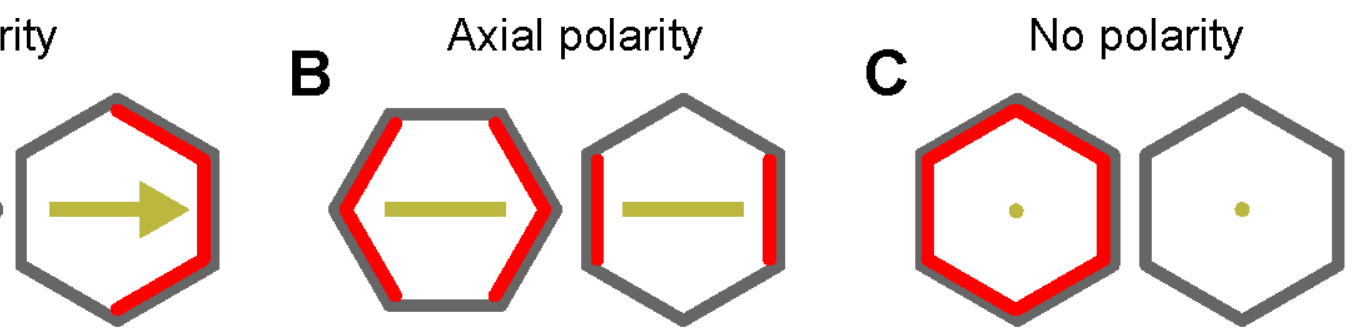

Axial and vectorial polarity appear axial
D

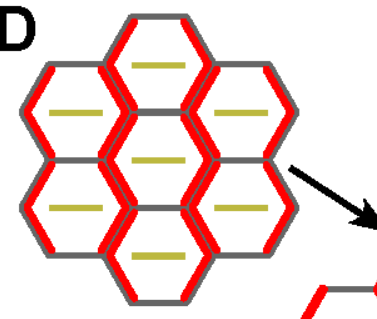

.

E
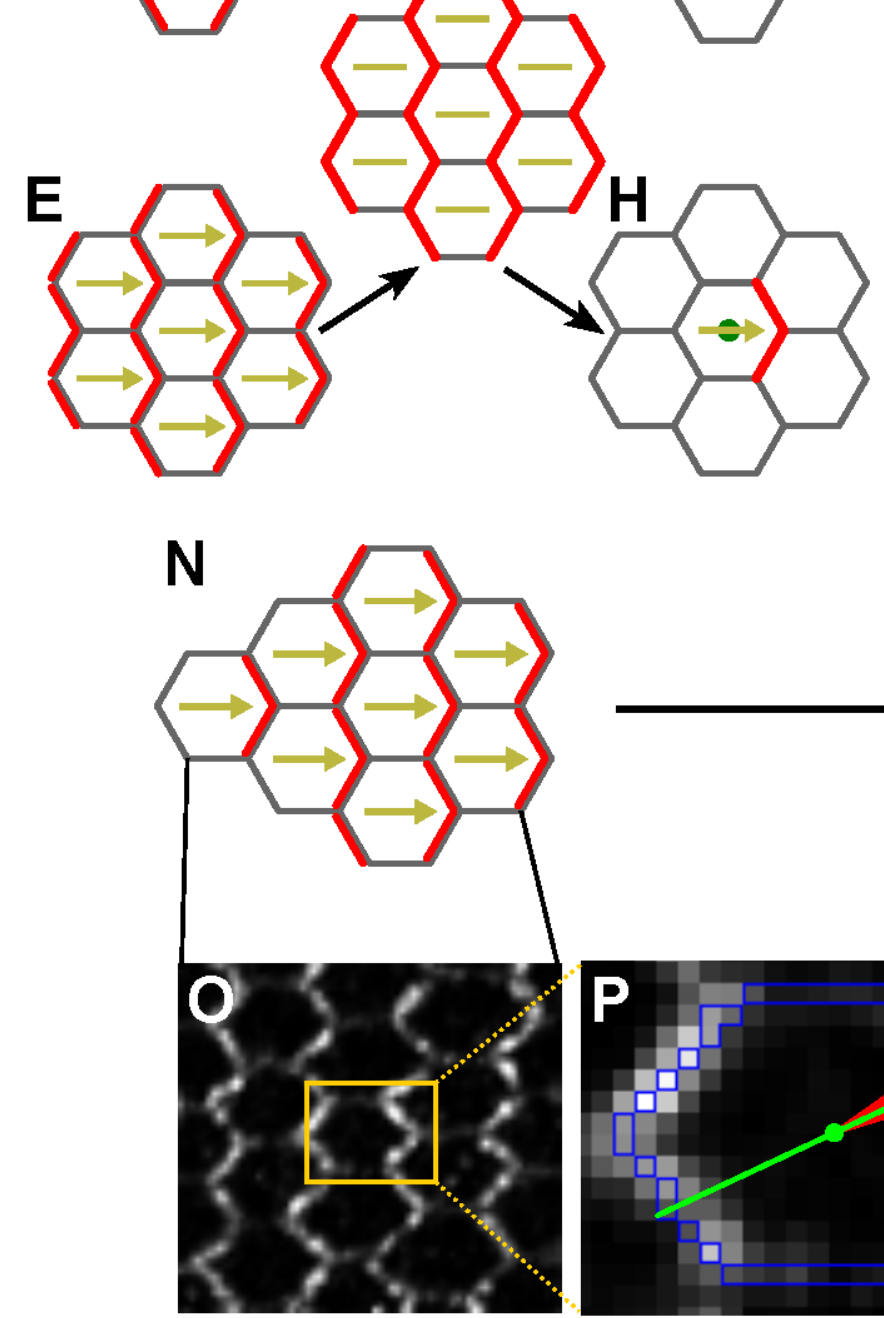

Mosaic staining can discrimanate between axial and vectorial polarity
Vectorial polarity may Mosaic staining can appear unpolarized reveal vectorial polarity

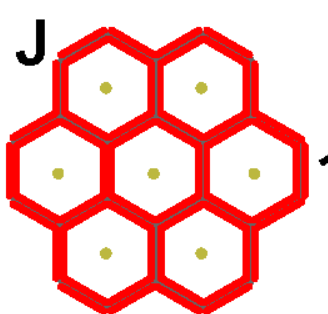

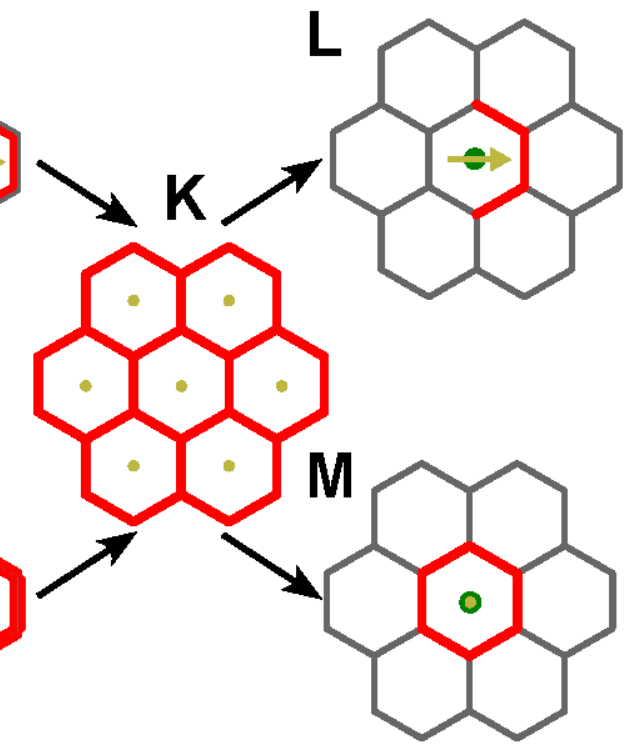

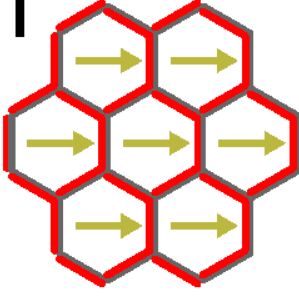

Q

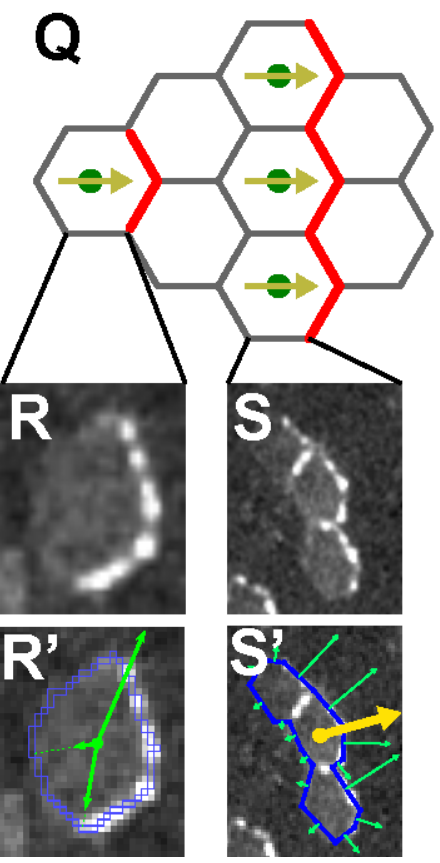




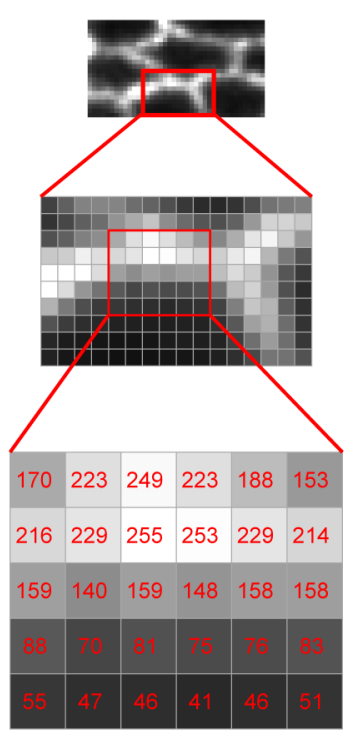

E

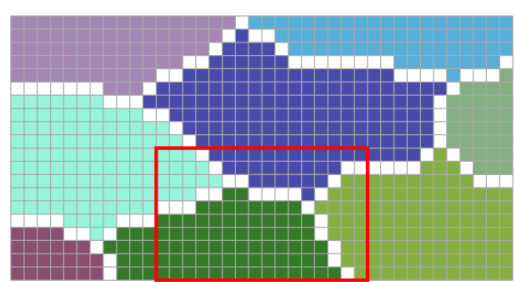

G

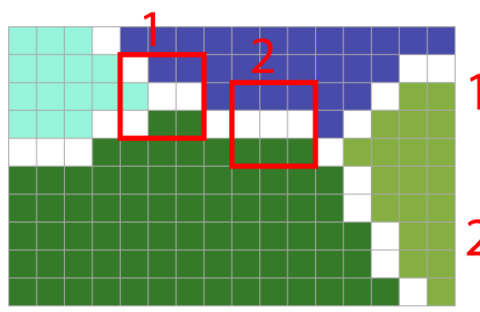

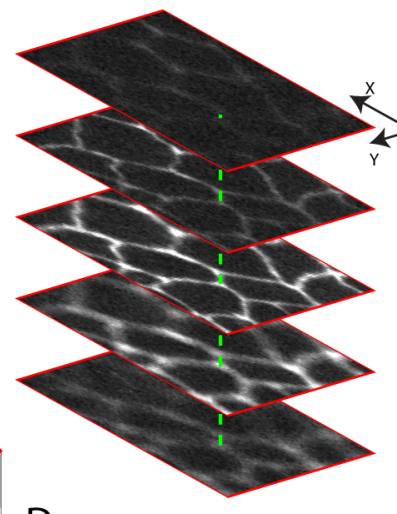

D

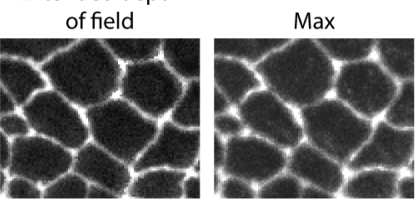

$\mathrm{F}$

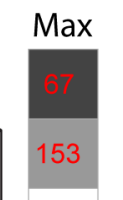

$255 *$

234
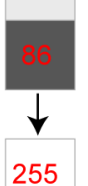

255

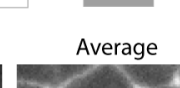

$\mathrm{D}^{\prime}$
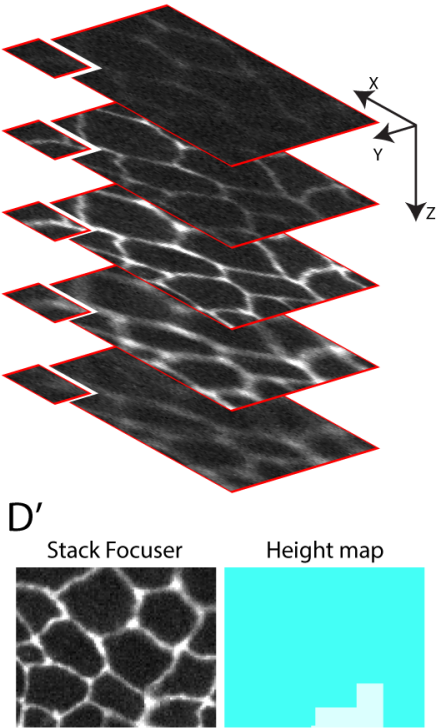

Height map
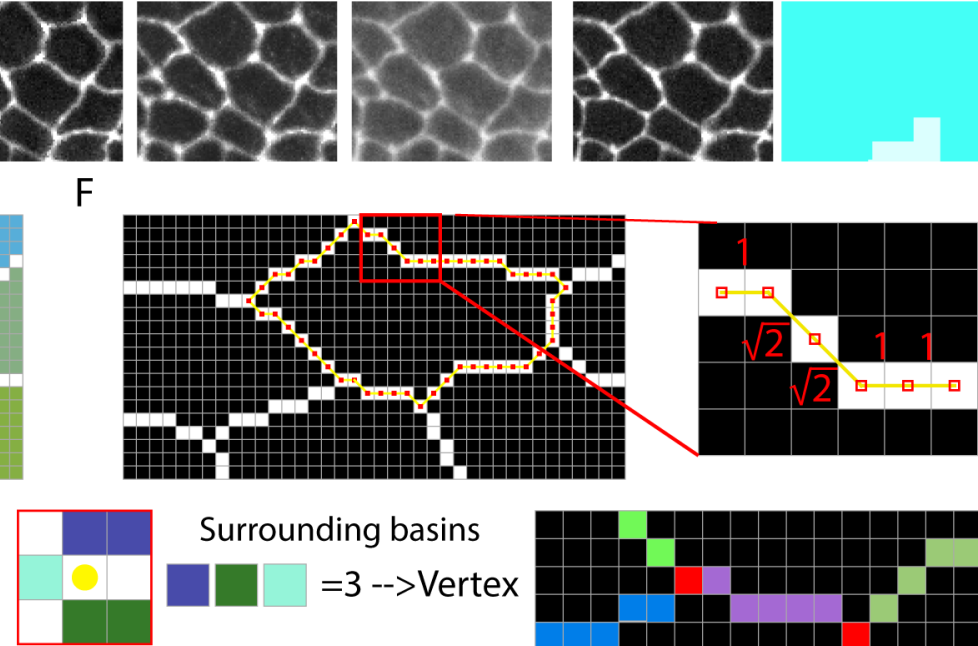

Surrounding basins

口 =3 -->Vertex

$2 \square=2->$ Bond

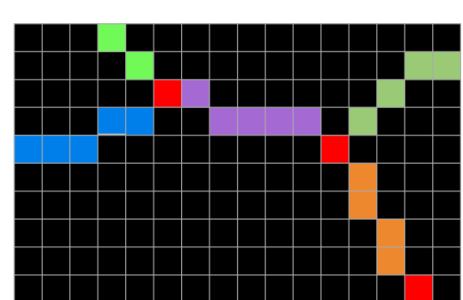


A cell shape change

T1 transition

cell division

T2 transition

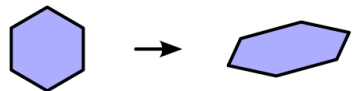

$\underset{d}{a}{ }_{d}^{c} b \rightarrow X \rightarrow{ }_{d}^{a}{ }_{d}^{c}$

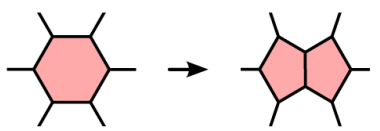

$\lambda \rightarrow$

B

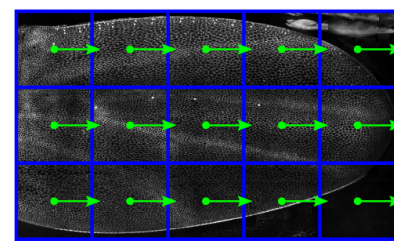

$\downarrow$ translation

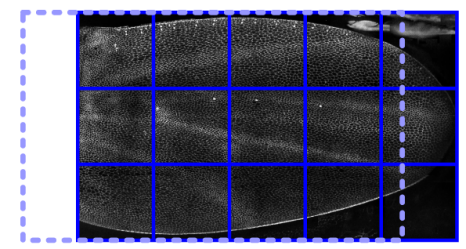

E isotropic deformation

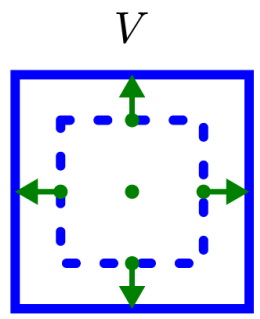

C
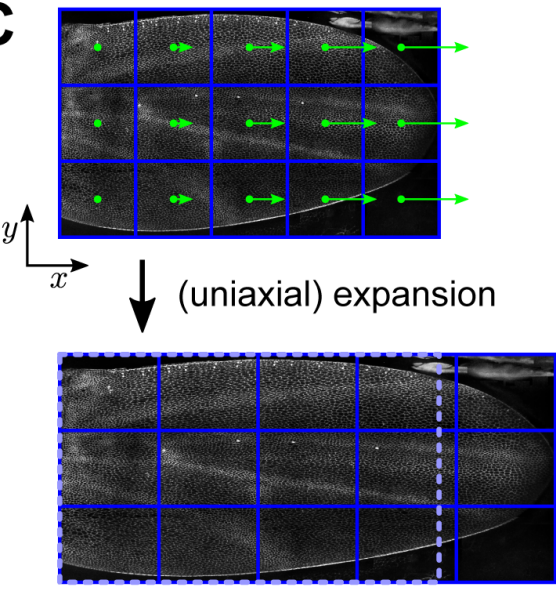

F anisotropic deformation $\tilde{V}_{x x}$

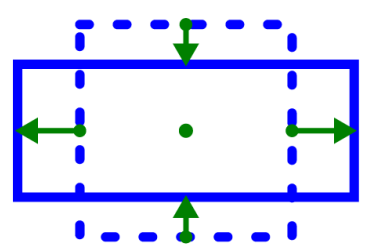

D
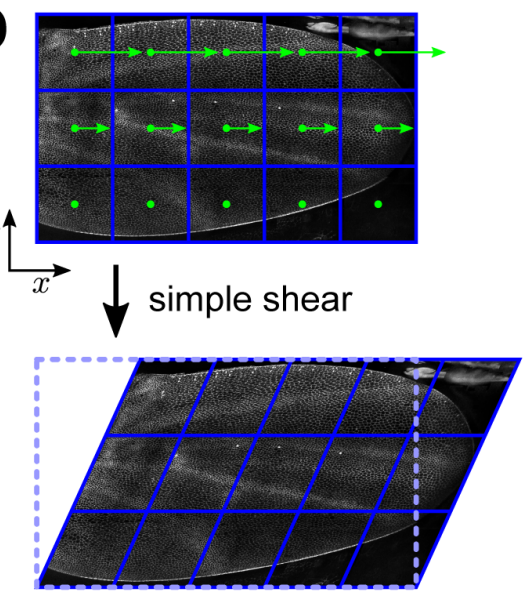

$\mathbf{G}$ rotation

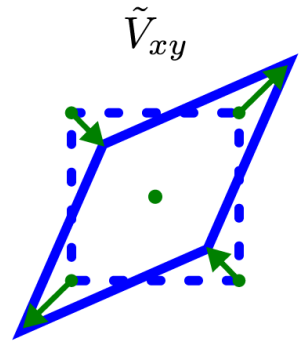

$\omega$

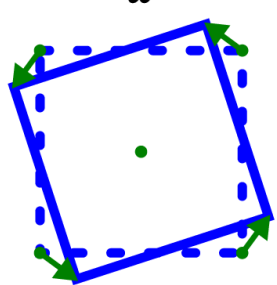

H

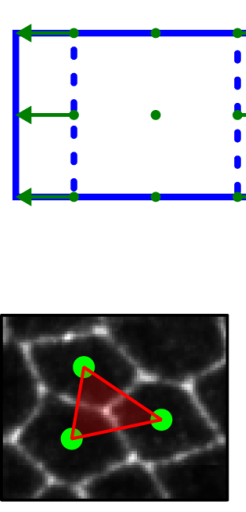

$\mathbf{L}$

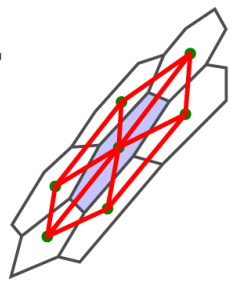

K

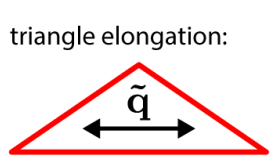

$M$

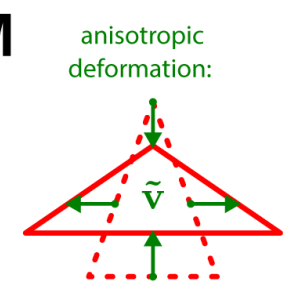

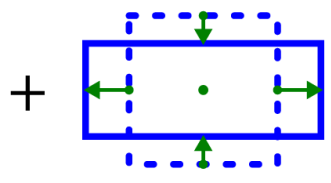

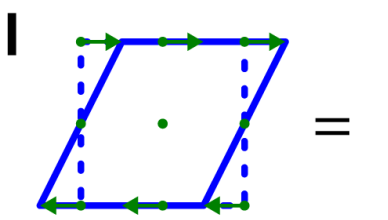

Triangle Method

$\mathbf{N}$

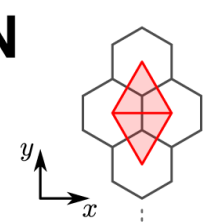

0

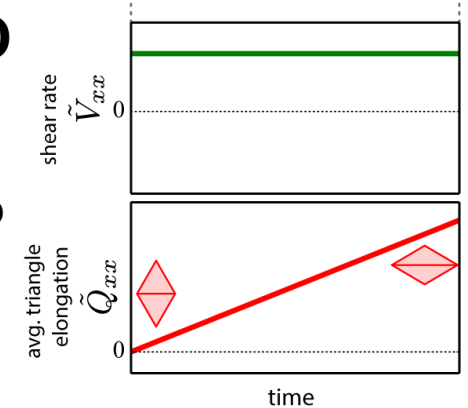

Q

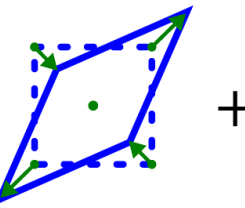

$+4$

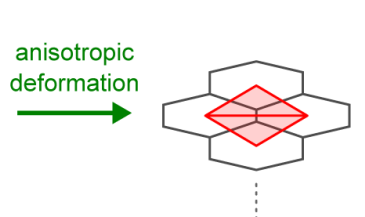

$\mathbf{R}$

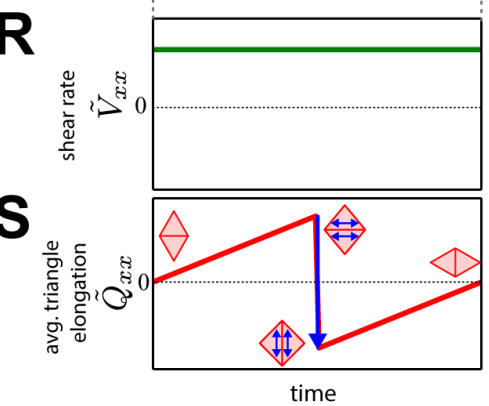

